\title{
Lentinan Supplementation Protects the Gut-Liver Axis and Prevents Steatohepatitis: The Role of Gut Microbiota Involved
}

\begin{abstract}
Xiaoying Yang ${ }^{1 \dagger}$, Mingxuan Zheng ${ }^{1 \dagger}$, Menglu Zhou ${ }^{1 \dagger}$, Limian Zhou ${ }^{1 \dagger}$, Xing Ge ${ }^{1}$, Ning Pang ${ }^{2}$, Hongchun $\mathrm{Li}^{3,4}$, Xiangyang $\mathrm{Li}^{1}$, Mengdi $\mathrm{Li}^{1}$, Jun Zhang ${ }^{5}$, Xu-Feng Huang ${ }^{6}$, Kuiyang Zheng ${ }^{1}$ and Yinghua $Y^{1,6 *}$

1 Jiangsu Key Laboratory of Immunity and Metabolism, Department of Pathogen Biology and Immunology, Xuzhou Medical University, Xuzhou, China, ${ }^{2}$ Tianjin Third Central Hospital, Tianjin, China, ${ }^{3}$ Medical Technology Institute, Xuzhou Medical University, Xuzhou, China, ${ }^{4}$ Department of Laboratory Medicine, Affiliated Hospital of Xuzhou Medical University, Xuzhou, China, ${ }^{5}$ Affiliated Hospital of Liaoning University of Traditional Chinese Medicine, Shenyang, China, ${ }^{6}$ School of Medicine, Illawarra Health and Medical Research Institute (IHMRI), University of Wollongong, Wollongong, NSW, Australia
\end{abstract}

\section{OPEN ACCESS}

Edited by:

Xi Ma

China Agricultural University, China

Reviewed by:

Peixin Fan,

University of Florida, United States Michael Müller,

University of East Anglia,

United Kingdom

*Correspondence:

Yinghua Yu

yinghua@uow.edu.au

tThese authors have contributed equally to this work and share first authorship

Specialty section

This article was submitted to Nutrition and Microbes, a section of the journal

Frontiers in Nutrition

Received: 28 October 2021 Accepted: 20 December 2021

Published: 20 January 2022

Citation:

Yang X, Zheng M, Zhou M, Zhou L,

Ge X, Pang N, Li H, Li X, Li M,

Zhang J, Huang $X-F$, Zheng $K$ and Yu Y (2022) Lentinan Supplementation

Protects the Gut-Liver Axis and Prevents Steatohepatitis: The Role of Gut Microbiota Involved. Front. Nutr. 8:803691 doi: 10.3389/fnut.2021.80369
The microbiota-gut-liver axis has emerged as an important player in developing nonalcoholic steatohepatitis (NASH), a type of nonalcoholic fatty liver disease (NAFLD). Higher mushroom intake is negatively associated with the prevalence of NAFLD. This study examined whether lentinan, an active ingredient in mushrooms, could improve NAFLD and gut microbiota dysbiosis in NAFLD mice induced by a high-fat (HF) diet. Dietary lentinan supplementation for 15 weeks significantly improved gut microbiota dysbiosis in HF mice, evidenced by increased the abundance of phylum Actinobacteria and decreased phylum Proteobacteria and Epsilonbacteraeota. Moreover, lentinan improved intestinal barrier integrity and characterized by enhancing intestinal tight junction proteins, restoring intestinal redox balance, and reducing serum lipopolysaccharide (LPS). In the liver, lentinan attenuated HF diet-induced steatohepatitis, alteration of inflammation-insulin (NFkB-PTP1B-Akt-GSK3 $\beta$ ) signaling molecules, and dysregulation of metabolism and immune response genes. Importantly, the antihepatic inflammation effects of lentinan were associated with improved gut microbiota dysbiosis in the treated animals, since the Spearman's correlation analysis showed that hepatic LPS-binding protein and receptor (Lbp and TIr4) and pro- and antiinflammatory cytokine expression were significantly correlated with the abundance of gut microbiota of phylum Proteobacteria, Epsilonbacteraeota and Actinobacteria Therefore, lentinan supplementation may be used to mitigate NAFLD by modulating the microbiota-gut-liver axis.

Keywords: mushroom, NAFLD, gut-liver axis, oxidative stress, gut microbiota, $\beta$-glucan

\section{INTRODUCTION}

Nonalcoholic fatty liver disease (NAFLD), one of the most common liver diseases worldwide, affects up to $30 \%$ of the adult population (1). Nonalcoholic steatohepatitis (NASH), a more severe form of NAFLD, is defined by the presence of steatosis with inflammation and progressive fibrosis, ultimately leading to cirrhosis or hepatocellular carcinoma (2). Recently, evidence for cross talk 
among the gut microbiota, the liver, the immune system, and energy metabolism has emerged, indicating that the microbiota is an important player in the development of NASH (3).

Compelling evidence links NASH with the alteration of the gut microbiome and intestinal barrier integrity. For example, the diversity and composition of gut microbiota significantly alter in the animal model and patients with $\mathrm{NASH}(4,5)$. The tight junction proteins, including zonula occludens-1 (ZO1) and occludin, decrease in the proximal small intestine of NASH mice (6). The gut integrity is disrupted by a redox imbalance in the intestine (7-10). Reactive oxygen species (ROS), such as nitric oxide (NO) and its generating enzyme inducible NO synthase (iNOS), induce the dysfunction and loss of tight junction of intestinal epithelial cells (7-9). In contrast, antioxidant enzymes, such as haem oxygenase 1 (HO1 ), NAD $(\mathrm{P}) \mathrm{H}$ quinone dehydrogenase 1 (NQO1), and glutamatecysteine ligase catalytic subunit (Gclc), play important roles in maintaining intestinal barrier integrity (10). The transcription factor of these antioxidant enzymes, nuclear factor erythroid 2related factor 2 (Nrf2), ameliorates intestinal barrier dysfunction induced by lipopolysaccharide (LPS, present in the outer membrane of gram-negative bacteria) (11). Therefore, gut microbiota dysbiosis and redox imbalance may disrupt gut integrity and lead to overtranslating the gut bacteria-derived toxin, such as LPS, into the port vein, thereby promoting inflammatory responses in the liver.

Lipopolysaccharide and its downstream pathway significantly contribute to hepatic inflammation in $\operatorname{NAFLD~}(12,13)$. LPS binds to its binding protein ( $\mathrm{Lbp}$ ), a $60-\mathrm{kDa}$ acute-phase protein, to elicit immune responses by presenting the LPS to toll-like receptor 4 (Tlr4, important cell surface pattern receptor) (14, 15). Activation of Tlr4 subsequently induces proinflammatory responses, including the activation of nuclear factor kappa $\mathrm{B}$ $(\mathrm{NF} \kappa \mathrm{B})$ and the increase in proinflammatory cytokines (TNF $\alpha$, IL-6, and IL-1 $\beta$ ) expression $(14,15)$. NFKB is also a transcription factor that increases protein tyrosine phosphatase $1 \mathrm{~B}$ (PTP1B, encoded by the ptpn 1 gene) (16), leading to insulin resistance. It is reported that patients with NASH have upregulation of hepatic Tlr4 mRNA and increased serum LPS (hyperendotoxinemia) (17). Therefore, the alteration of the gut-liver axis mediated by gut microbiota metabolite, LPS, may play a critical role in the development and progression of NASH.

Mushrooms have been used as food and medicinal resources for millennia (18). A clinical study has reported that higher mushroom intake was negatively associated with the NAFLD prevalence among adults (19). It is speculative that the active ingredient in mushrooms, such as $\beta$-glucan, contributes to

\footnotetext{
Abbreviations: AUC, area under curve; DEGs, differentially expressed genes; Gclc, glutamate-cysteine ligase catalytic subunit; GTT, glucose tolerance test; HF, highfat diet; HFL, high-fat diet supplemented with lentinan; HO-1, haem oxygenase 1 ; iNOS, inducible NO synthase; INSR, insulin receptor; Lbp, lipopolysaccharidebinding protein; LF, low-fat diet; LPS, lipopolysaccharide; Mcp1, monocyte chemoattractant protein-1; NAFLD, nonalcoholic fatty liver; NFKB, nuclear factor kappa B; NQO1, NAD(P)H quinone dehydrogenase 1; Nrf2, nuclear factor-E2related factor 2; PTP1B, protein tyrosine phosphatase $1 \mathrm{~B}$; ROS, reactive oxygen species; Tlr4, toll-like receptor 4; TNF $\alpha$, tumor necrosis factor alpha; ZO-1: zonula occludens- 1 .
}

the beneficial effect. Lentinan from shiitake mushroom, a $\beta(1,3) / \beta(1,6)$-glucan, has a $\beta(1,3)$ backbone branched with short $\beta(1,6)$-linked side chains. In comparison, the $\beta$-glucans in cereal are primarily in $\beta(1,4)$ linkages separating shorter stretches of $\beta(1,3)$ structure. The mixed-linkage $\beta(1,3) / \beta(1,4)$-glucan (MLG) of cereal is the specific hydrolysis by the MLG utilization locus (MLGUL) of some gut microbiota, such as Bacteroides ovatus (20), resulting in compositional and functional shifts in the gut microbiota $(21,22)$. Recently, it has been reported that lentinan, $\beta(1,3) / \beta(1,6)$-glucans of mushroom, alleviates LPSinduced intestinal injury through modulating the composition and metabolites of intestinal microbiota in piglet (23). However, lentinan's potential effects and mechanism on the gut-liver axis in NAFLD represent a gap in the current research.

In this study, using the high-fat (HF) diet-induced NASH mouse model, we examined the effects of lentinan on gut microbiota, gut redox balance, and tight junction proteins, and also lipid deposition, NFКB/PTP1B signaling of endotoxin LPS, macrophage infiltration, and transcriptome regulation of immune and metabolic response in the liver.

\section{MATERIALS AND METHODS}

\section{Materials}

Lentinan (Figure 1A) of purity $>98 \%$ was purchased from Yuanye Biological Technology Co., Ltd (Shanghai, China). Details of sources of antibodies and other reagents used are shown in the Supplementary Data.

\section{Animals and Treatment}

Male C57BL/6J mice aged 9 weeks were purchased from the Experimental Animal Center of Xuzhou Medical University [Xuzhou, China, SCXK (Su) 2015-0009], housed, and maintained in a 12-h light/dark photoperiod with unrestricted water and food. All animal care and experiments were carried out under protocols approved by the ethics committee of Xuzhou Medical University. After habituation to the laboratory environment for 1 week, the mice were randomly divided into four groups $(N=10$ per group): (1) mice fed a low-fat (LF) diet ( $5 \%$ fat by weight) as a control (LF) group; (2) mice fed the LF diet supplemented with lentinan (500 $\mathrm{mg} / \mathrm{Kg}$ in diet) as the LFL group; (3) mice receiving the HF diet (31.5\% fat by weight) as the HF group; and (4) mice fed the HF diet supplemented with lentinan $(500 \mathrm{mg} / \mathrm{Kg}$ in diet) as the HFL group. The detailed information of LF and HF diets is in Supplementary Table S1. The consumption of $500 \mathrm{mg} / \mathrm{Kg}$ lentinan in the diet is equivalent to dose of $\sim 60 \mathrm{mg} / \mathrm{kg}$ body weight for mice, which was decided according to an effective dose of $\beta$-glucan at $30 \mathrm{mg} / \mathrm{kg}$ in reduction of blood glucose in rats (24) and transferred by dose translation formula between mice and rats (25). Lentinan (98\%) was purchased from Yuanye Biological Technology Co., Ltd (Shanghai, China). Mice were administered the three diets for 15 weeks. Body weight and food intake were measured on the last day of each week. After 13 weeks of feeding, an intraperitoneal glucose tolerance test (GTT) was performed as detailed below. Mice were then euthanized after 15 weeks of feeding. The data of body weight, energy intake, liver weight, and GTT were collected from 10 mice per group. After the mice 


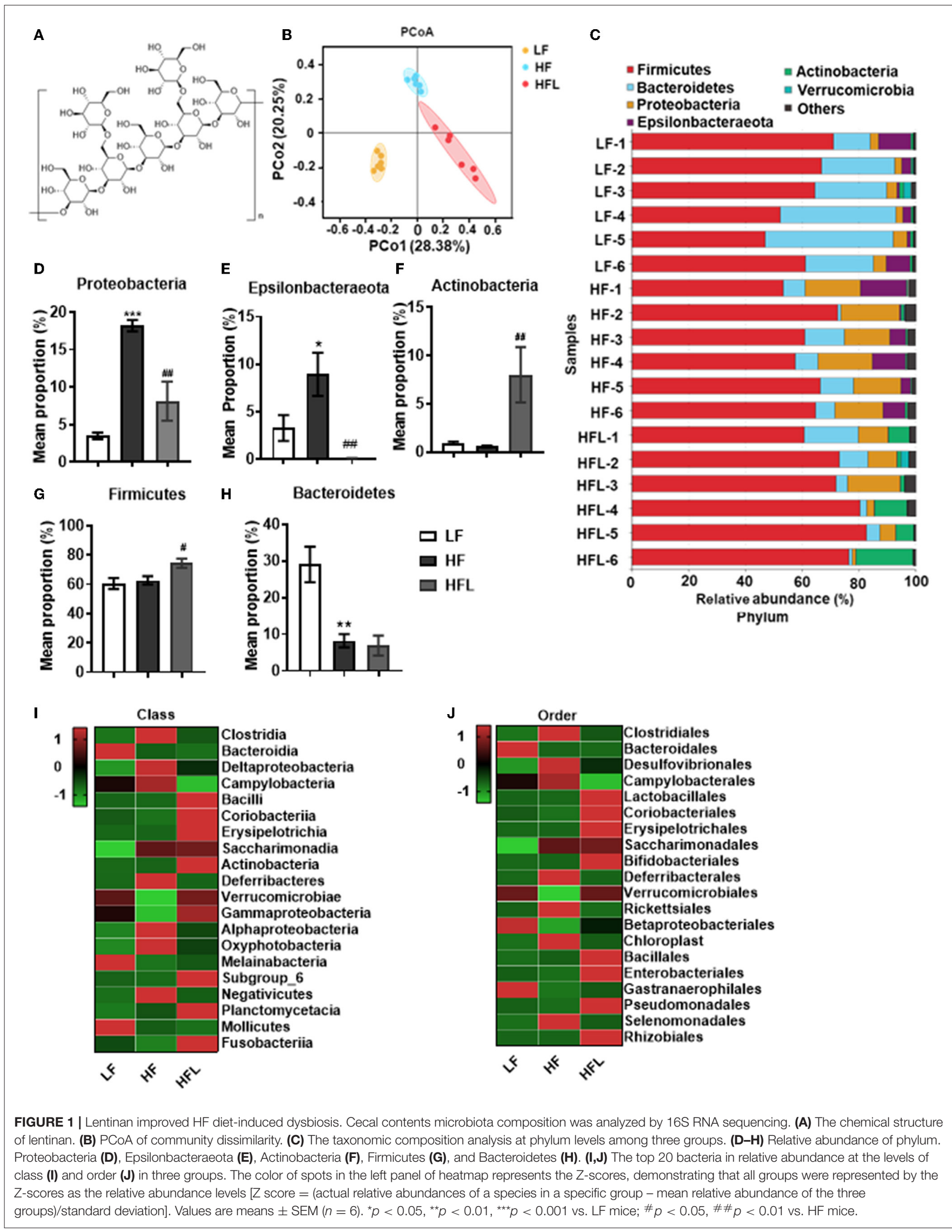


were sacrificed ( $n=6$ mice per group), blood was collected to detect serum LPS levels; fresh tissues of liver and intestine were collected to perform qPCR and western blot; cecum contents were collected for gut microbiota analysis. In addition, after mice were perfused with paraformaldehyde ( $n=4$ mice per group), the liver and intestinal tissues were collected for oil red $\mathrm{O}$ staining, Hematoxylin and eosin ( $\mathrm{H} \& \mathrm{E})$ staining, immunohistochemistry, and immunofluorescence.

\section{Glucose Tolerance Test}

The GTT was performed as we have previously described (26). The detailed methods are described in the Supplementary Data.

\section{RNA-Sequencing Analysis}

RNA sample preparation and sequencing: Total RNA was extracted from livers of mice in LF, HF, and HFL groups (three mice in each group) using TRIzol reagent kit (Invitrogen, Carlsbad, California, USA). Subsequently, the mRNA was enriched by oligo (dT) beads. The enriched mRNA was fragmented with fragmentation buffer and reverse-transcribed into cDNA with random primers. Second-strand cDNA was synthesized by DNA polymerase I, RNase H, dNTP, and buffer. The cDNA fragments were purified with a QiaQuick PCR extraction kit (Qiagen 28104), end repaired, poly(A) added, and ligated to Illumina sequencing adapters. The ligation products were size selected by agarose gel electrophoresis, PCR amplified and sequenced using Illumina HiSeq 4000 by CapitalBio Technology Co., Ltd (China).

Gene-level differential expression analyses: Differential expression genes (DEGs) analysis for mRNA was performed using R package edge R. DEGs with $\mid \log 2($ fold change) $\mid$ value $>1$ and $p<0.05$, considered as significantly modulated (27), were retained for further analysis. The KEGG pathway enrichment analysis was performed for DEGs using the Database for Annotation, Visualization, and Integrated Discovery (DAVID) (28). The protein-protein interaction (PPI) network of the proteins encoded by the DEGs was searched using the STRING database and Cytoscape software (29).

\section{LPS Determination}

The serum concentration of LPS was determined using a chromogenic end-point TAL kit. The absorbance was measured at $545 \mathrm{~nm}$ using a spectrophotometer, with measurable concentrations ranging from 0.1 to $1.0 \mathrm{EU} / \mathrm{ml}$. All samples for LPS measurements were performed in duplicate.

\section{Oil Red O Staining}

Oil red $\mathrm{O}$ staining was used to examine hepatic lipid accumulation as described previously (30). The detailed methods are provided in the Supplementary Data.

\section{Hematoxylin and Eosin Staining}

The H\&E staining detection and histological assessment are described in the Supplementary Data.

\section{Immunohistochemistry}

The immunohistochemistry methods for hepatic F4/80 and intestinal iNOS detection and quantification are provided in the Supplementary Data.

\section{Immunofluorescence}

Jejunum segments were immunostained as described previously (31). The detailed methods are described in the Supplementary Data.

\section{Western Blotting}

Western blot assays were performed as described previously (32). The methods are depicted in the Supplementary Data.

\section{Quantitative RT-PCR Analysis}

Total RNA was extracted with RNA Isolator Total RNA Extraction Reagent (Vazyme, China) from the liver and jejunum, before reverse-transcription to cDNA using a high-capacity cDNA reverse transcription kit (Takara, Japan). qPCR was performed using the TransStart $\AA$ Top Green qPCR SuperMix (TransGen, China) and determined on a real-time PCR detection system (Roche LightCycler480, Switzerland). The mRNA levels for specific genes were calculated using the formula $2(-\Delta \Delta \mathrm{Ct})$ and normalized by $\beta$-actin mRNA levels. All primers are listed in Supplementary Table S2.

\section{Gut Microbiota Analysis}

DNA extraction, PCR amplification, and 16S rRNA gene sequencing: After 15 weeks of feeding, the cecum contents of mice were collected. Microbial DNA was extracted using the HiPure Stool DNA Kit (Magen, Guangzhou, China) according to the protocol recommended by the manufacturer. V3-V4 region of 16s rRNA genes was amplified by PCR with the primers 341-F, 5'-CCT ACGGGNGGCWGCAG-3' and 806-R, 5'-GGACTACHVGGG TATCTAAT-3' (33), and the amplification procedure was as follows: Initial denaturation at $94^{\circ} \mathrm{C}$ for $2 \mathrm{~min}$, followed by denaturation at $98^{\circ} \mathrm{C}$ for $10 \mathrm{~s}$, annealing at $65^{\circ} \mathrm{C}$ for $30 \mathrm{~s}$, and extension at $68^{\circ} \mathrm{C}$ for $30 \mathrm{~s}$. This round was repeated for 30 cycles, followed by a final extension at $68^{\circ} \mathrm{C}$ for $5 \mathrm{~min}$. PCRs were performed in triplicate, and the reaction system was composed of $5 \mathrm{ml}$ of $10 \times \mathrm{KOD}$ buffer, $5 \mathrm{ml}$ of $2 \mathrm{mM}$ dNTPs, $3 \mathrm{ml}$ of $25 \mathrm{mM} \mathrm{MgSO} 4,1.5 \mathrm{ml}$ of each primer $(10 \mathrm{mM}), 1 \mathrm{ml}$ of KOD polymerase, and $100 \mathrm{ng}$ of template DNA, with $50 \mathrm{ml}$ in total. After amplification, the products were purified using the AxyPrep DNA Gel Extraction Kit (Axygen Biosciences, Union City, CA, USA) and quantified using ABI StepOnePlus Real-Time PCR System (Life Technologies, Foster City, USA). Purified products were pooled in equimolar and paired-end sequenced (PE250) on an Illumina platform according to the standard protocols.

Sequence data processing: To get high-quality clean reads, raw reads containing more than $10 \%$ of unknown nucleotides-(N) and reads with $<60 \%$ of bases with a quality value (Q-value) $>20$ were removed using FASTP (version 0.18.0) (34). Pairedend clean reads were merged as raw tags using FLSAH (version 1.2.11) (35) with a minimum overlap of $10 \mathrm{bp}$ and mismatch error rates of $2 \%$. Noisy sequences of raw tags were filtered 
by QIIME (version 1.9.1) (36) pipeline under specific filtering conditions (37) to obtain high-quality clean tags. The filtering conditions were as follows: (1) break raw tags from the first lowquality base site where the number of bases in the continuous low-quality value (the default quality threshold is $\leq 3$ ) reaches the set length (the default length is three); (2) then, filter tags whose continuous high-quality base length was $<75 \%$ of the tag length. The clean tags were searched against the reference database (http://drive5.com/uchime/uchime_download.html) to perform reference-based chimera checking using the UCHIME algorithm. After chimeric tags were removed, the final effective tags were used for further analysis.

Bioinformatic analysis: The clean tags were clustered into operational taxonomic units (OTUs) of $\geq 97 \%$ similarity using UPARSE (version 9.2.64) pipeline (38). The $\alpha$-diversity indices evaluating gut microbial community richness (the Ace and Chaol estimators) and community diversity (the Shannon estimator) were calculated using Mothur (39). Principal coordinate analysis (PCoA) based on Bray-Curtis distance and permutational multivariate analysis of variance (PERMANOVA) was performed to compare the global microbiota composition after the intervention in each group at phylum, genus, and OTU levels, respectively. The difference in abundant taxa was detected by the linear discriminant analysis (LDA) effect size (LEfSe) algorithm, which emphasizes statistical significance, biological consistency, and effect relevance (40). Differences with $\log 10 \mathrm{LDA}$ scores (absolute values) $>3.0$ and $p<0.05$ were considered significant (41). The functional potential of the gut microbial communities was estimated by the PICRUSt12 algorithm (42). In univariate analysis of gut microbiota and predicted KEGG biochemical pathways in each group, a paired $t$-test or a Wilcoxon matched-pairs test was adopted and $p$-values were adjusted for multiple comparisons using the BenjaminiHochberg false discovery rate.

\section{Statistical Analysis}

Statistical analysis was performed using SPSS (version 20, IBM Corporation, Chicago, IL, USA). All data were tested for normality by applying the Shapiro-Wilk normality test. If normality was given, the one-way analysis of variance (ANOVA) was performed, followed by the post-hoc, Tukey's test for comparisons among the groups. Correlations between gut bacterial abundance and serum LPS and its binding protein and receptor in the liver and also hepatic proinflammatory and antiinflammatory cytokines were calculated using Spearman's correlation analysis. Differences were considered significant when $p<0.05$ and marked with ${ }^{*}$ or ${ }^{\#}\left({ }^{*} p<0.05,{ }^{* *} p<0.01\right.$, ${ }^{* * *} p<0.001,{ }^{\#} p<0.05,{ }^{\# \#} p<0.01$, $\left.{ }^{\# \# \#} p<0.001\right)$. Values are expressed as the mean \pm standard error of means (SEM).

\section{RESULTS}

\section{Dietary Lentinan Supplementation Improved Gut Microbiota Dysbiosis in HF Mice}

Gut microbiota dysbiosis plays an important role in NAFLD onset and progression $(43,44)$. Here, by $16 \mathrm{~S}$ rRNA gene sequencing analysis, we investigated the gut microbiota after the HF diet with or without lentinan (Figure 1A) supplementation for 15 weeks. Principal coordinate analysis (PCoA) of microbiota showed a clear cluster separation among the LF, HF, and HFL groups (Figure 1B), whereas the LF diet-fed mice with lentinan supplementation had a similar cluster with the LF mice (Supplementary Figure S1A). The HF mice showed a decrease in gut microbial community richness (the Chaol and Ace estimators) and community diversity (the Shannon estimator) (all $p<0.05$, Supplementary Figures S1B-D), whereas lentinan prevented the reduction of gut microbial community richness (all $p<0.05$, Supplementary Figures S1B,C). Among the LF, $\mathrm{HF}$, and HFL groups, the difference in the relative abundance of microbiota at phylum was shown in Supplementary Figure S1E. Specifically, the gut microbiota of these three groups was mainly dominated by Firmicutes, Bacteroidetes, Proteobacteria, Epsilonbacteraeota, and Actinobacteria at the phylum level (Figure 1C), in which Proteobacteria and Epsilonbacteraeota increased (both $p<0.05$, Figures 1D,E), and Bacteroidetes decreased in the HF group ( $p<0.05$, Figure 1H). Importantly, lentinan supplementation reduced the abundance of Proteobacteria and Epsilonbacteraeota and increased Actinobacteria and Firmicutes (all $p<0.05$, Figures 1D-G and Supplementary Figure S1E). At the class level, Clostridia, Bacteroidia, Deltaproteobacteria, Campylobacteria, and Bacilli were dominant bacterial (Supplementary Figure S1F), in which Clostridia, Deltaproteobacteria, and Campylobacteria were deceased in the HF mice with lentinan supplementation compared with that of the HF group (Figure 1I). At the order level, Clostridiales, Bacteroidales, Desulfovibrionales, Campylobacterales, and Lactobacillales accounted for the majority of gut microbiota (Supplementary Figure S1G). Compared with HF mice, supplementation of lentinan decreased the relative abundance of Clostridiales, Desulfovibrionales, and Campylobacterales and increased Lactobacillales (Figure 1J).

Furthermore, in HF mice, the low taxa belonging to phylum Proteobacteria, including class Deltaproteobacteria, order Desulfovibrionales, and family Desulfovibrionaceae, were all significantly increased (Figures 2A-C and Supplementary Figures S1F-H). Phylum Epsilonbacteraeota's lower taxa, including class Campylobacteria, order Campylobacterales, family Helicobacteraceae, and genus Helicobacter, were significantly increased in $\mathrm{HF}$ mice (Figures 2D-G and Supplementary Figures S1F-I). Importantly, lentinan prevented HF-induced alterations in these microbiota belonging to the phylum Proteobacteria and Epsilonbacteraeota. Notably, bacteria belonging to Actinobacteria, order Bifidobacteriales, family Bifidobacteriaceae, and genus Bifidobacterium were elevated significantly in HF mice with lentinan supplementation (Figures 2H-J and Supplementary Figures S1G-I). LDA effect size (LEfSe) showed that the lower taxa bacteria of the phylum Epsilonbacteraeota, such as order Campylobacterales, class Campylobacteria, family Helicobacteraceae, and genus Helicobacter, belonging to phylum Proteobacteria were enriched in the HF group (Figure 2K). In HFL mice, bacteria belonging to order Bifidobacteriales, family Bifidobacteriaceae, and genus Bifidobacterium were elevated. In addition, genus 

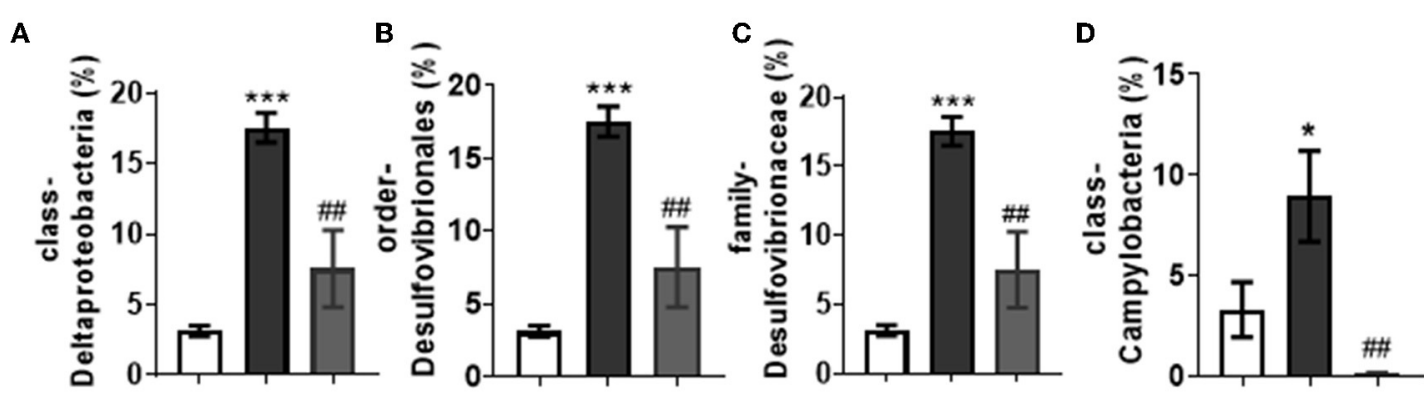

E

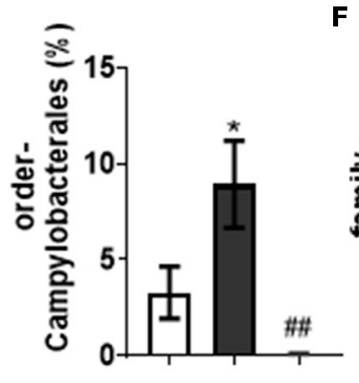

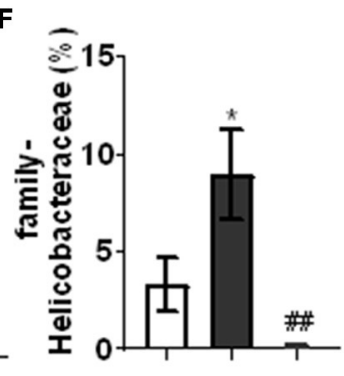
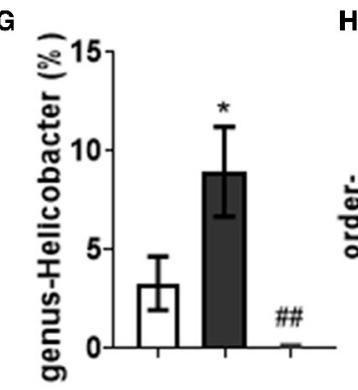

H
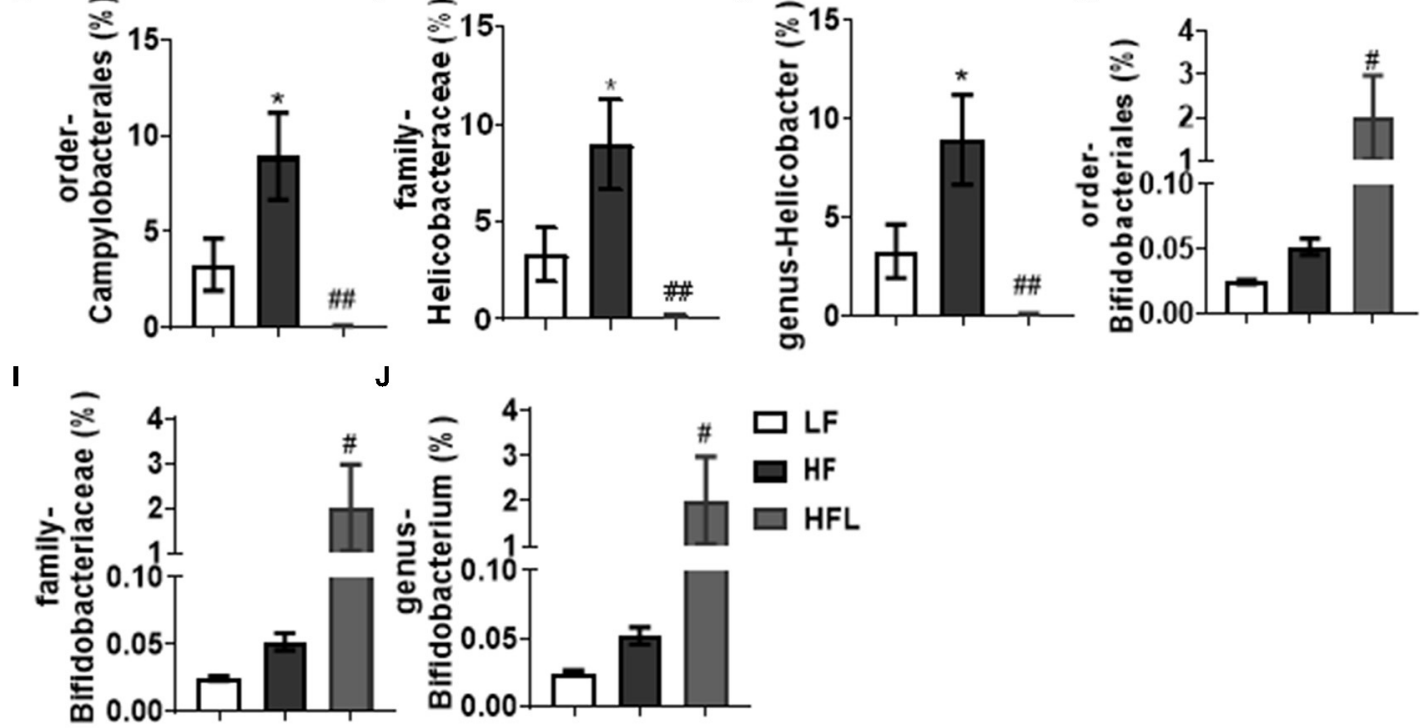

,

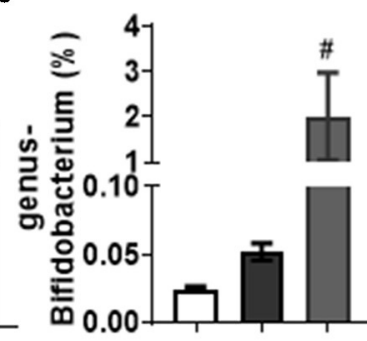

$\mathbf{K}$

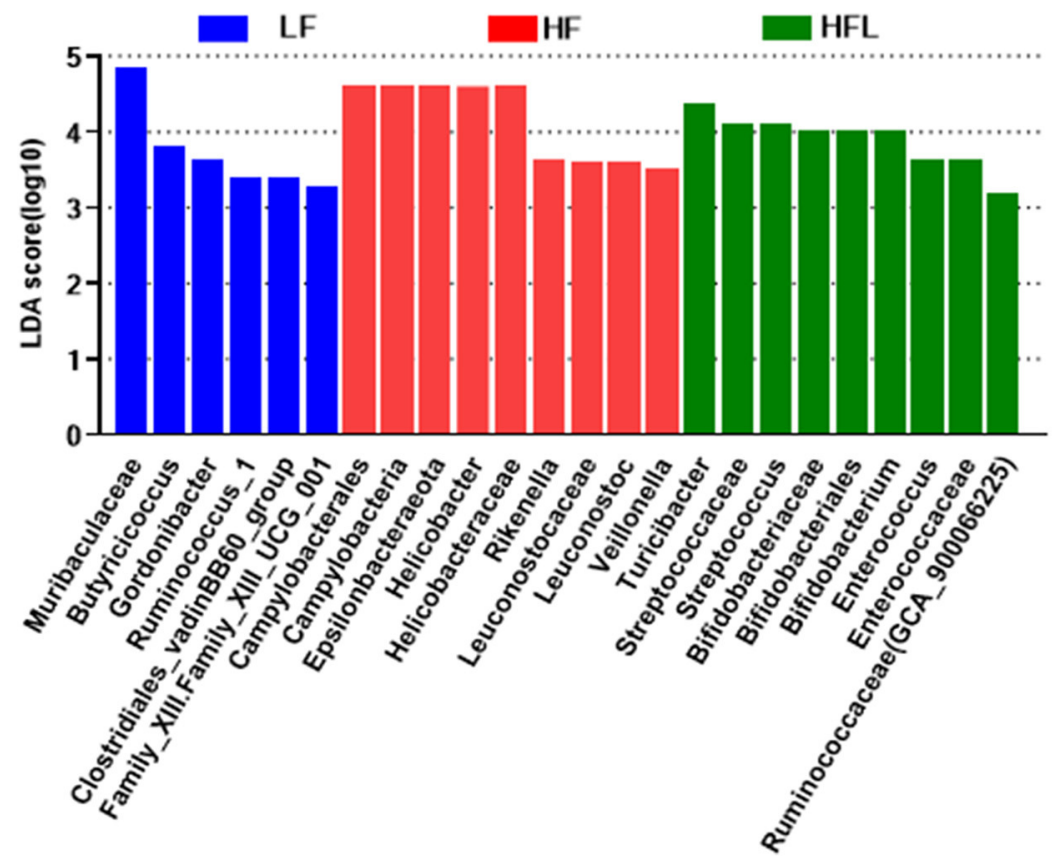

FIGURE 2 | Lentinan restored the HF-induced compositional shift of gut microbial community. (A-K) Comparison of the representative taxonomic abundance of class Deltaproteobacteria (A), order Desulfovibrionales (B), family Desulfovibrionaceae (C), class Campylobacteria (D), order Campylobacterales (E), family

Helicobacteraceae (F), genus Helicobacter (G), order Bifidobacteriales (H), family Bifidobacteriaceae (I), and genus Bifidobacterium (J). (K) LEfSe results of mice gut microbiomes. Values are means $\pm \operatorname{SEM}(n=6) .{ }^{*} p<0.05,{ }^{\star \star *} p<0.001$ vs. LF mice; ${ }^{\#} p<0.05$, ${ }^{\# \#} p<0.01$ vs. HF mice. 
TABLE 1 | Predicted KEGG functional pathway differences at level 2 inferred from 16S rRNA gene sequence using PICRUSt after LF, HF and HFL diet.

\begin{tabular}{|c|c|c|c|c|c|c|}
\hline \multicolumn{2}{|c|}{ KO functional categories } & \multirow[t]{2}{*}{ LF } & \multirow[t]{2}{*}{ HF } & \multirow[t]{2}{*}{ HFL } & \multirow{2}{*}{$\frac{\text { LF vs. HF }}{p \text {-value }}$} & \multirow{2}{*}{$\begin{array}{l}\text { HF vs. HFL } \\
p \text {-value }\end{array}$} \\
\hline Level One & Level Two & & & & & \\
\hline \multirow[t]{7}{*}{ Metabolism } & Carbohydrate Metabolism & $12.062 \pm 0.232$ & $11.862 \pm 0.335$ & $12.522 \pm 0.228$ & - & 0.001 \\
\hline & Amino Acid Metabolism & $10.842 \pm 0.321$ & $10.431 \pm 0.181$ & $10.421 \pm 0.264$ & 0.016 & - \\
\hline & Nucleotide Metabolism & $4.562 \pm 0.153$ & $4.408 \pm 0.12$ & $4.608 \pm 0.188$ & - & 0.042 \\
\hline & Enzyme Families & $2.569 \pm 0.025$ & $2.476 \pm 0.052$ & $2.528 \pm 0.063$ & 0.005 & - \\
\hline & Glycan Biosynthesis and Metabolism & $2.683 \pm 0.217$ & $2.414 \pm 0.151$ & $2.108 \pm 0.165$ & 0.020 & 0.010 \\
\hline & Metabolism of Other Amino Acids & $1.655 \pm 0.033$ & $1.551 \pm 0.025$ & $1.639 \pm 0.075$ & 0.003 & 0.008 \\
\hline & Biosynthesis of Other Secondary Metabolites & $1.037 \pm 0.044$ & $0.947 \pm 0.035$ & $0.982 \pm 0.043$ & 0.002 & - \\
\hline Genetic Information Processing & Transcription & $3.424 \pm 0.117$ & $3.483 \pm 0.144$ & $3.631 \pm 0.077$ & - & 0.044 \\
\hline \multirow[t]{2}{*}{ Environmental Information Processing } & Signal Transduction & $2.139 \pm 0.247$ & $2.44 \pm 0.193$ & $2.04 \pm 0.254$ & 0.041 & 0.010 \\
\hline & Signaling Molecules and Interaction & $0.194 \pm 0.003$ & $0.179 \pm 0.011$ & $0.212 \pm 0.03$ & - & 0.007 \\
\hline \multirow[t]{2}{*}{ Cellular Processes } & Cell Motility & $3.629 \pm 0.992$ & $4.453 \pm 0.691$ & $3.155 \pm 0.716$ & - & 0.014 \\
\hline & Transport and Catabolism & $0.415 \pm 0.045$ & $0.335 \pm 0.034$ & $0.306 \pm 0.047$ & 0.005 & - \\
\hline Human Diseases & Metabolic Diseases & $0.106 \pm 0.011$ & $0.094 \pm 0.004$ & $0.103 \pm 0.008$ & - & 0.025 \\
\hline \multirow[t]{6}{*}{ Organismal Systems } & Environmental Adaptation & $0.201 \pm 0.011$ & $0.221 \pm 0.010$ & $0.199 \pm 0.007$ & 0.002 & 0.001 \\
\hline & Nervous System & $0.111 \pm 0.004$ & $0.103 \pm 0.002$ & $0.109 \pm 0.004$ & 0.001 & 0.010 \\
\hline & Immune System & $0.105 \pm 0.012$ & $0.094 \pm 0.006$ & $0.092 \pm 0.006$ & 0.044 & - \\
\hline & Digestive System & $0.023 \pm 0.006$ & $0.015 \pm 0.003$ & $0.014 \pm 0.004$ & 0.016 & - \\
\hline & Excretory System & $0.02 \pm 0.005$ & $0.011 \pm 0.004$ & $0.012 \pm 0.004$ & 0.003 & - \\
\hline & Circulatory System & $0.009 \pm 0.008$ & $0.015 \pm 0.011$ & $0 \pm 0.001$ & - & 0.007 \\
\hline
\end{tabular}

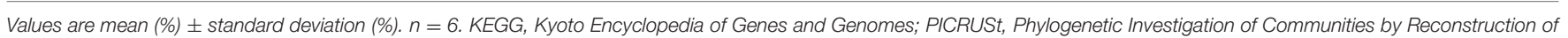
Unobserved States; KO, KEGG Ortholog; LF, low-fat diet; HF, high-fat diet; HFL, HF diet supplemented with lentinan.

Turicibacter, family Streptococcaceae, genus Streptococcus, genus Enterococcus, family Enterococcaceae, and genus Ruminococcaceae (GCA_900066225) were also increased in HFL mice. Moreover, KEGG functional orthologs predicted potential functional interactions between the gut microbiota and host among the LF, HF, and HFL group in 19 functional orthologs at level two (Table 1). Totally, 12 functional orthologs were significantly altered in the HF group compared with the LF group. Compared with the HF group, the lentinan supplementation was associated with microbial functional shifts in 12 functional orthologs, including carbohydrate metabolism, nucleotide metabolism, glycan biosynthesis and metabolism, metabolism of other amino acids, transcription, signal transduction, and so on.

\section{Dietary Lentinan Supplementation Improved Hyperendotoxinemia, Tight Junction Proteins, and Redox Imbalance in the Jejunum of HF Mice}

Gut microbiota alteration can promote the endotoxinemia and alteration of intestinal barrier integrity (45). After identifying the capacity of lentinan to prevent gut dysbiosis, we next examined the serum LPS and the tight junction proteins, occludin and ZO1 in the jejunum. The serum LPS level was significantly increased in the HF fed mice, whereas lentinan inhibited the elevation of serum LPS in the mice fed by HF diet, but not by LF diet ( $p$ $<0.01$, Figure 3A). Meanwhile, the mRNA expressions of the tight junction proteins, occludin and ZO-1, were significantly lower in the jejunum of HF diet-fed mice compared with LF and HFL groups (all $p<0.05$, Figure 3B). In line with these findings in transcription level, the protein levels of the tight junction proteins were significantly increased in the mice with lentinan supplementation (all $p<0.05$, Figure 3C). The HF mice showed reduced immunofluorescence staining of both occludin and ZO-1, whereas clear and uniform positive staining of the tight junction proteins was found in the epithelium of jejunum in LF and HFL groups (Figure 3D), suggesting that intestinal barrier integrity was improved by lentinan supplementation.

Given that redox imbalance is the major factor for intestinal barrier dysfunction (7-10), we next investigated the effects of lentinan supplementation on antioxidants and oxidative stress markers in the jejunum. The expression of antioxidants Nrf2, HO-1, NQO1, and Gclc was decreased in the HF group, whereas lentinan supplementation significantly prevented the decrease in antioxidants induced by the HF diet (all $p<0.05$, Figures 3E,F). Moreover, lentinan significantly inhibited the HF diet-mediated increments of iNOS protein (all $p<0.05$, Figures $3 E-G$ ). These findings suggest lentinan supplementation attenuated redox imbalance in the intestine of HF mice.

\section{Dietary Lentinan Supplementation Prevented HF Diet-Induced Hepatosteatosis and Hepatic Inflammation}

Following lentinan improving gut microbiota, redox balance, and tight junction proteins, we further examined the effects of lentinan on hepatic lipid deposition and macrophage infiltration. 


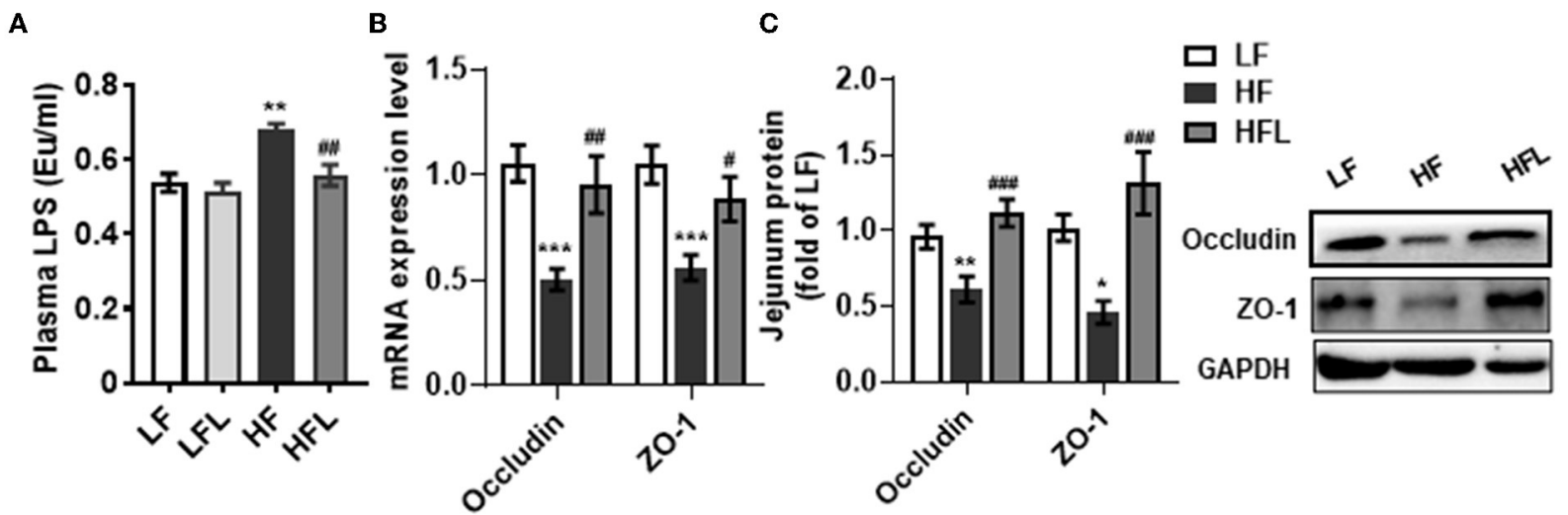

D

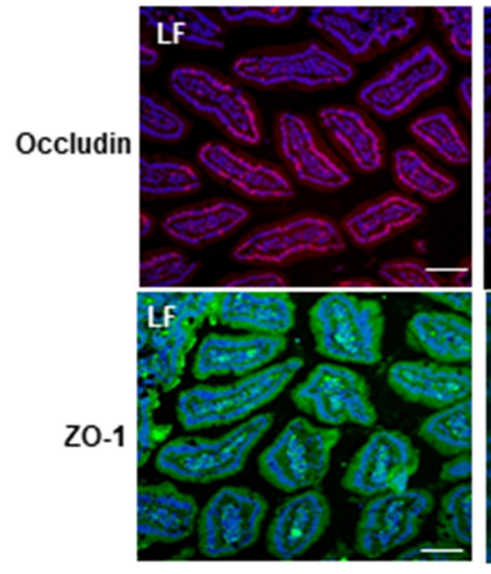

E
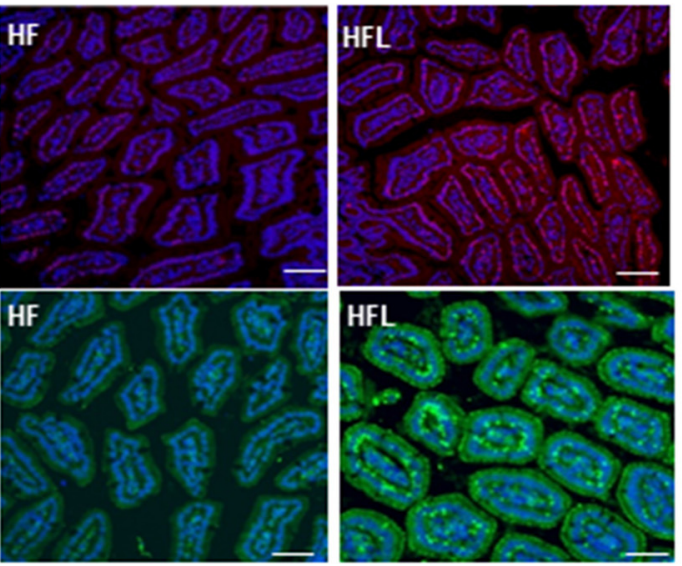

$\mathbf{F}$

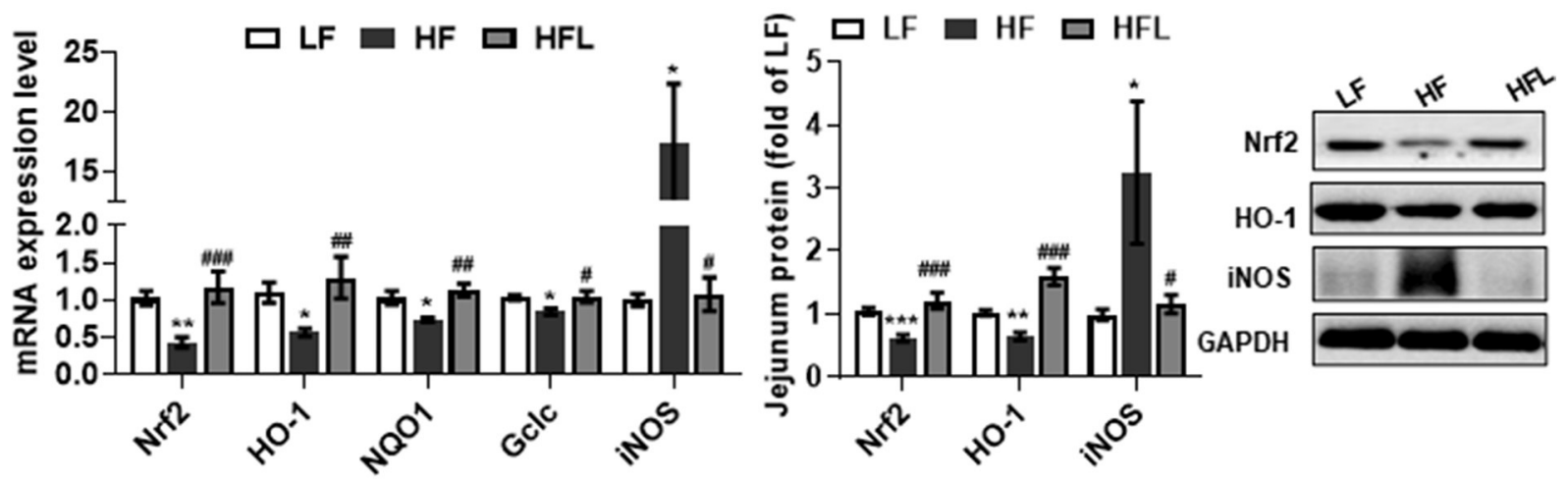

G
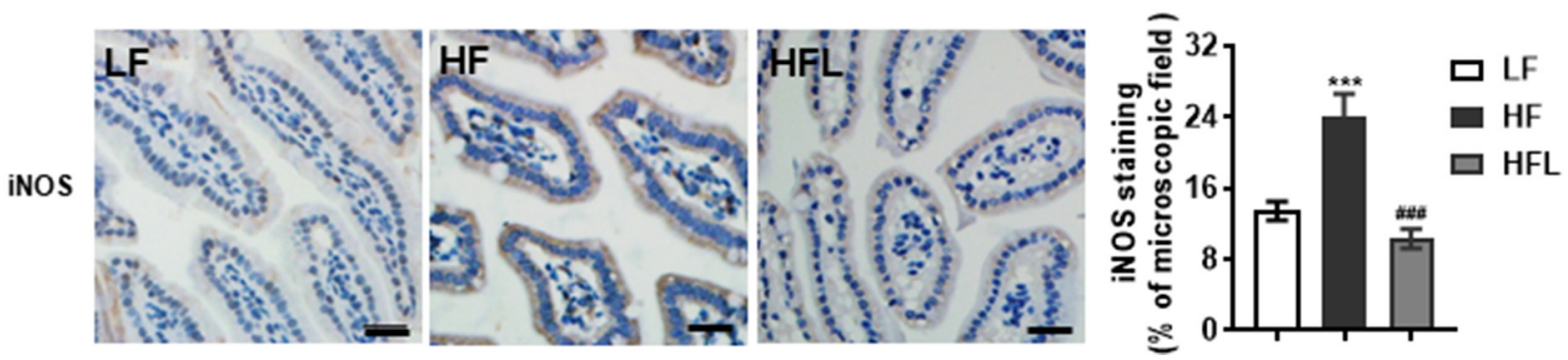

FIGURE 3 | Lentinan improved intestinal barrier integrity and oxidative stress in the jejunum in HF mice. (A) Serum LPS levels. (B) The mRNA levels of occludin and ZO-1 in the jejunum. (C) Protein levels of occludin and ZO-1 in the jejunum. (D) Immunofluorescence staining of occludin and ZO-1 proteins in the jejunum sections. (E) The mRNA levels of Nrf2, HO-1, NQO1, Gclc, and iNOS in the jejunum. (F) Protein levels of Nrf2, HO-1, and iNOS in the jejunum. (G) iNOS protein staining of 
FIGURE 3 | jejunum sections and quantification of iNOS protein after staining. Values are means \pm SEM $(n=6)$. ${ }^{*} p<0.05,{ }^{* \star} p<0.01,{ }^{\star \star *} p<0.001$ vs. LF mice; ${ }^{\#} p$ $<0.05, \# \# p<0.01, \# \# \# p<0.001$ vs. HF mice. Scale bar: $50 \mu \mathrm{M}$.

With oil red $\mathrm{O}$ and $\mathrm{H} \& \mathrm{E}$ staining, the hepatocytes of the HF group contained larger cytoplasmic lipid droplets $(p<$ 0.001 , Figures 4A,B) and were enlarged with ballooning $(p<$ 0.001 , Figures 4 C,D), compared with LF mice. These alterations in hepatic cellular morphology were significantly prevented by lentinan supplementation. Moreover, we found that the number of F4/80-positive cells (macrophage marker) was higher in HF mice compared with LF mice and HFL mice (both $p<0.05$, Figures 4E,F), indicating that lentinan suppressed macrophage infiltration induced by HF diet. Importantly, lentinan supplementation reduced the mRNA expression of macrophage-related gene CD68 and CD11c (the marker of proinflammatory M1 type macrophages), but increased the mRNA expression of CD206 (the marker of antiinflammatory M2 type macrophages) (all $p<0.05$, Figure 4G), suggesting that the M1 macrophage polarization was inhibited by lentinan. Furthermore, lentinan supplementation inhibited the mRNA of the proinflammatory cytokines and chemokines, TNF $\alpha$, IL-1 $\beta$, IL-6, and monocyte chemoattractant protein-1 (Mcp1) compared with HF mice (all $p<0.05$, Figure $4 \mathbf{H}$ ) and increased the expression of antiinflammatory cytokines, IL-10, and arginase I (Arg1) (both $p<0.05$, Figure 4H). Moreover, the mRNA expressions of Lbp and Tlr4 (the LPS-binding protein and cell surface pattern recognition receptors) were significantly higher in the liver of HF mice compared to LF mice and HFL mice ( $p<0.05$, Figure $4 \mathbf{H}$ ), which is consistent with the increase in serum LPS levels in the HF group described previously (Figure 3A).

The Spearman's correlation analysis was used to investigate the relationship between gut bacterial abundance and serum LPS and its binding protein and receptor in the liver and also hepatic proinflammatory and antiinflammatory cytokines (Figure 4I). Phylum Proteobacteria and its down taxa (class Deltaproteobacteria, order Desulfovibrionales, and family Desulfovibrionaceae), and also phylum Epsilonbacteraeota and its down taxa (class Campylobacteria, order Campylobacterales, family Helicobacteraceae and genus Helicobacter), were positively correlated with LPS, Lbp, Tlr4, and proinflammatory cytokines, whereas negatively correlated with antiinflammatory cytokines, IL-10, and Arg1. Phylum Actinobacteria and its down taxa (order Bifidobacteriales, family Bifidobacteriaceae, and genus Bifidobacterium) were negatively correlated with Tlr4 and positively correlated with IL-10. Therefore, these findings suggest that lentinan in attenuation of HF diet-induced hepatic inflammation was associated with the improvement of gut microbiota profile.

\section{Dietary Lentinan Supplementation Improved Glucose Intolerance and Hepatic NFкB-PTP1B-Akt-GSK3 $\beta$ Signaling Pathway in HF Mice}

Hepatic inflammation contributes to insulin resistance and abnormal glucose metabolism (46). Next, we determined whether lentinan could improve glucose metabolism. In the GTT test, lentinan decreased the blood glucose level at 60-, 90-, and 120 min time points (all $p<0.05$, Figure 5A). The glucose area under curve (AUC) was markedly higher in HF mice than in LF, LFL, and HFL mice (both $p<0.01$, Figure 5B). Consistently, mRNA expression of insulin receptor (INSR) was significantly lower in livers of HF mice than that in the other two groups (both $p<0.001$, Figure 5C). PTP1B is a mediator of the proinflammatory $(\mathrm{NF} \kappa \mathrm{B})$ signaling pathway in dysregulation of insulin (pAkt-pGSK3 $\beta$ ) signaling cascade (47). We found that lentinan significantly decreased the total and phosphorylated protein levels of NFKB subunit p65 and also PTP1B protein level in livers of HF mice (all $p<0.001$, Figure 5D). Furthermore, the phosphorylation levels of Akt and GSK3 $\beta$ significantly decreased in HF diet-fed mice, whereas lentinan reversed the reduction in these two insulin signaling molecules (both $p<0.05$, Figure 5D). These results showed that lentinan improved glucose intolerance and retrieved the insulin Akt-GSK3 $\beta$ signaling pathway in HF mice. In addition, we observed that after the HF diet for 15 weeks, the mice had significantly higher body weight, body weight gain, energy intake, and liver weight compared with LF dietfed mice, and lentinan supplementation prevented these changes (Supplementary Table S3). However, these metabolic indexes of LFL mice were not significantly altered compared with LF group (Supplementary Table S3).

\section{Dietary Lentinan Supplementation Modulated Hepatic Transcriptome Related to Metabolism and Immune Response}

To gain comprehensive profiles of lentinan supplementation on the hepatic metabolisms and inflammation at the transcriptome level, RNA-sequencing (RNA-seq) of liver tissues of $\mathrm{HF}$ and HFL groups was performed, followed by differentially expressed genes (DEGs) screening analysis. Consequently, lentinan supplementation modulated a total of 749 genes, including 408 upregulated genes and 341 downregulated genes (Figures 6A,B). Within the organismal system, 48 genes were significantly altered in the immune system, the most affected system (Figure 6C). In metabolism pathways, there were 146 genes altered, including 24 in lipid metabolism, 8 in glycan biosynthesis and metabolism, and 13 in carbohydrate metabolism. The top 20 KEGG pathways associated with DEGs are shown in Supplementary Table S4. Of these, the energy metabolism and immune response-related pathways were significantly affected in the liver of mice with lentinan supplementation, such as PI3K-Akt signaling pathway, metabolic pathways, arachidonic acid metabolism, and cytokine-cytokine receptor interaction. The PPI network was constructed to clarify the interaction of DEGs involved in hepatic lipid metabolism, carbohydrate metabolism, immune system, and PTP1B-Akt signaling affected by lentinan. The results demonstrated that the complex interaction network of immune and metabolism was significantly intervened by the lentinan supplementation (Figure 6D). The connectivity degree 


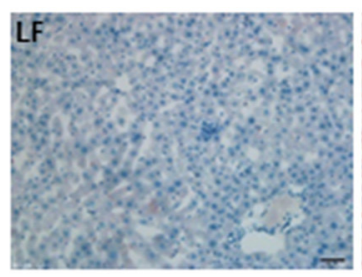

C

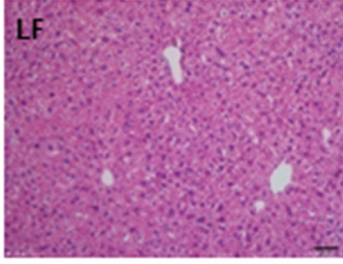

E
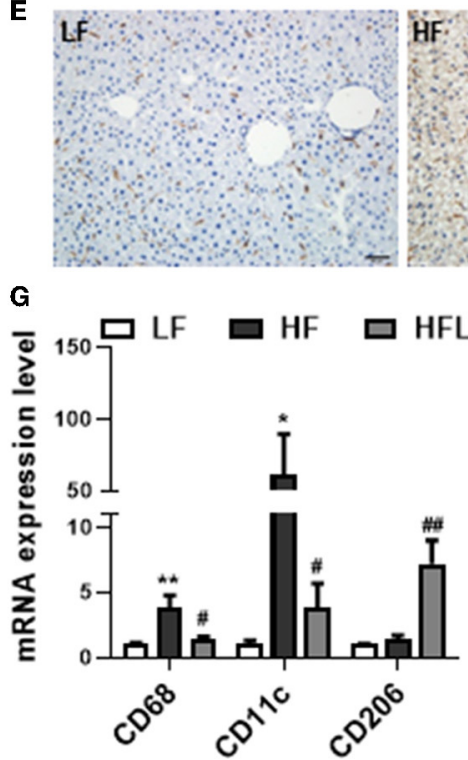

HF:
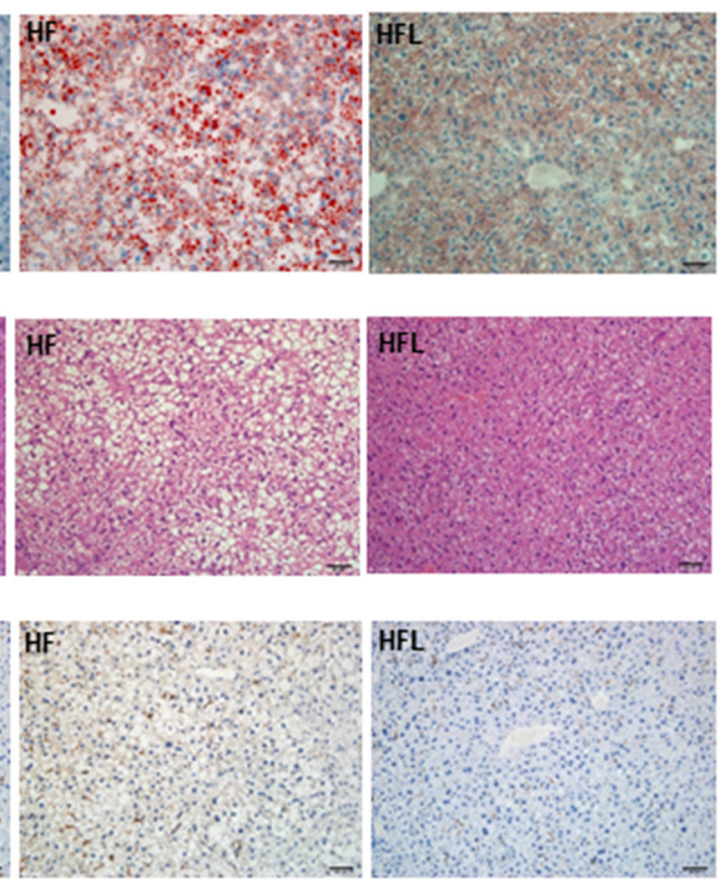

H
HFL

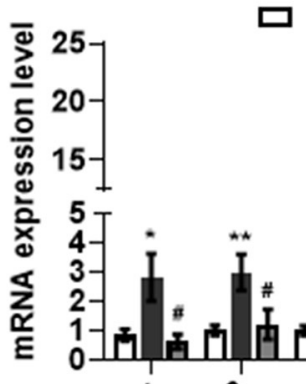

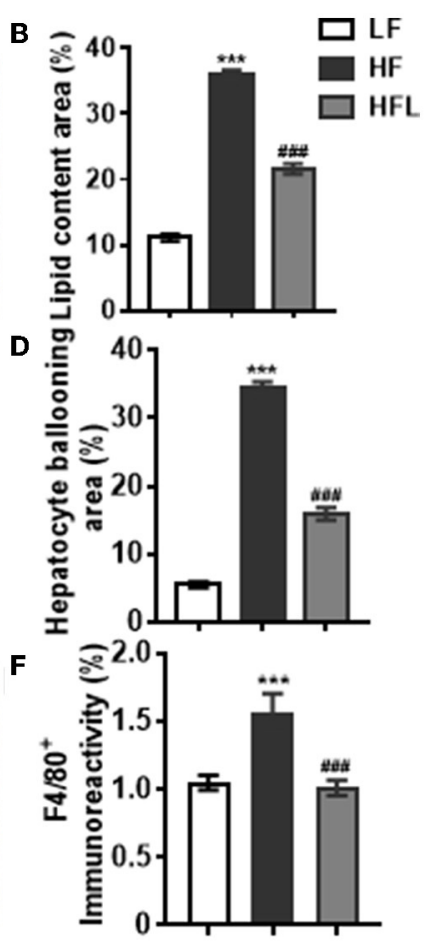

I

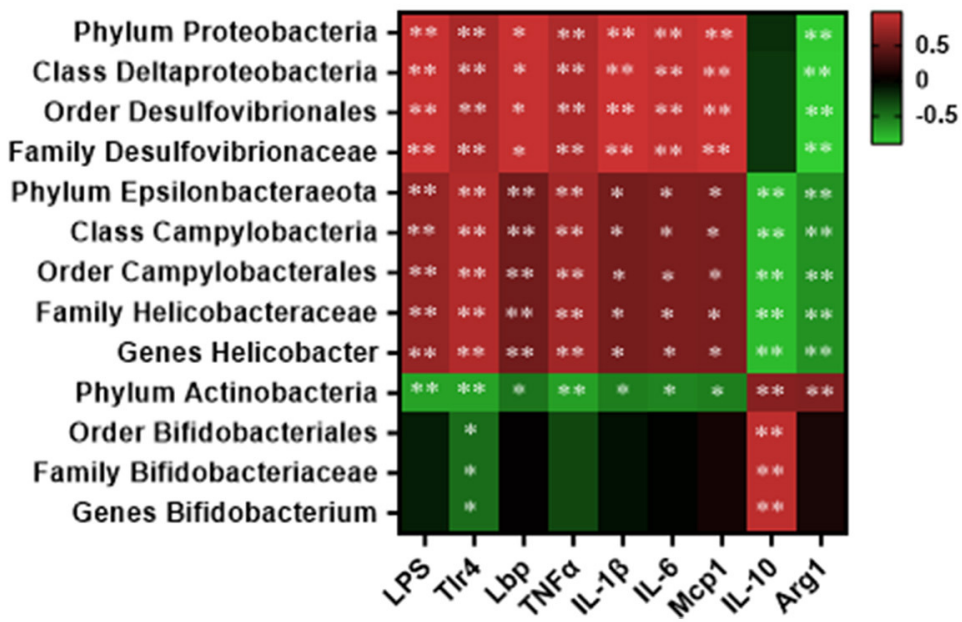

FIGURE 4 | Lentinan prevented HF diet-induced hepatic lipid deposition, macrophage infiltration, and hepatic inflammation. (A) Oil red O staining of liver sections. (B) Quantification of hepatic lipid area after Oil red O staining. (C) H\&E staining of liver sections. (D) Quantification of hepatic ballooning after H\&E staining. (E) F4/80 protein staining of liver sections. (F) Densitometric analysis of F4/80 staining. (G) CD68, CD11c, and CD206 mRNA levels in liver tissues of mice. (H) The mRNA levels 
FIGURE 4 | of TNF $\alpha$, IL-1 $\beta$, IL-6, Mcp1, IL-10, Arg1, Lbp, and TIr4 in liver tissues. (I) Correlation between specific gut microbiota, serum LPS, its binding protein and receptor in the liver, and hepatic proinflammatory and antiinflammatory cytokine expression. Values are means \pm SEM $(n=6) .{ }^{*} p<0.05,{ }^{* *} p<0.01,{ }^{* \star *} p<0.001$ vs. LF mice; $\# p<0.05, \# \# p<0.01$, \#\#\# $p<0.001$ vs. HF mice.

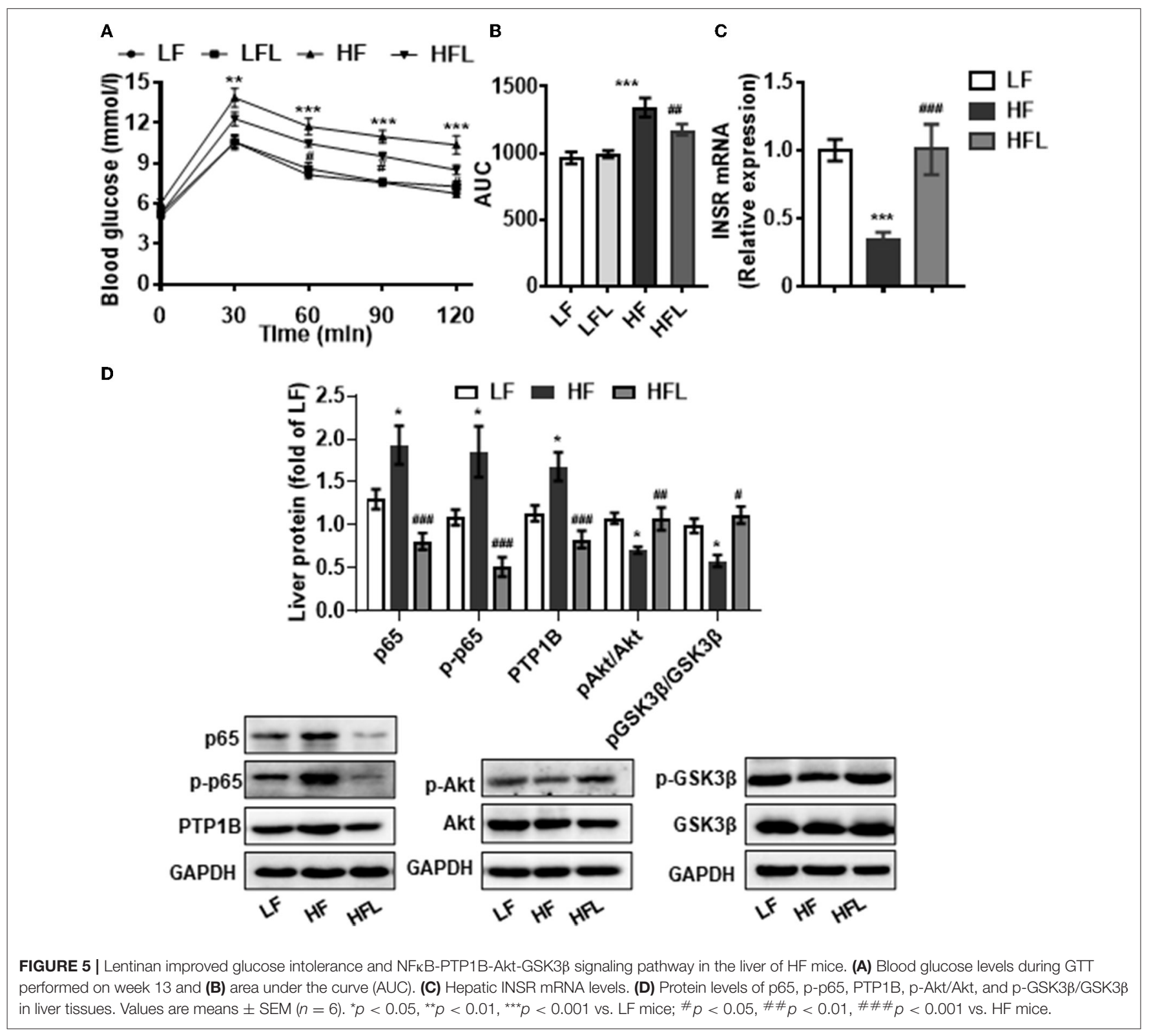

of PTP1B-Akt signaling with the PPI network was 38, indicating that PTP1B-Akt is a key node with a higher centrality value in the interaction network regulated by lentinan in HF diet-fed mice. Furthermore, we confirmed the mRNA expression of 10 DEGs by qPCR (Figure 6E).

\section{DISCUSSION}

In this study, we demonstrated that chronic dietary supplementation of lentinan attenuated gut microbiota alteration, intestinal tight junction deficit, and redox imbalance induced by a HF diet. In the liver, lentinan suppressed steatohepatitis and improved endotoxemia (LPS) and its downstream NFKB-PTP1B-Akt-GSK3 $\beta$ (inflammation-insulin) signaling pathway. Furthermore, Spearman's correlation analysis revealed that the gut phylum Proteobacteria, Epsilonbacteraeota, and Actinobacteria, and also their next level taxa, were correlated with LPS-related-binding protein and receptor, and inflammatory cytokines in the liver. Previous animal and epidemiological studies suggest that mushroom intake has beneficial effects for steatohepatitis and NALFD (48-50). For example, in a large population-based study (a cross-sectional 
A

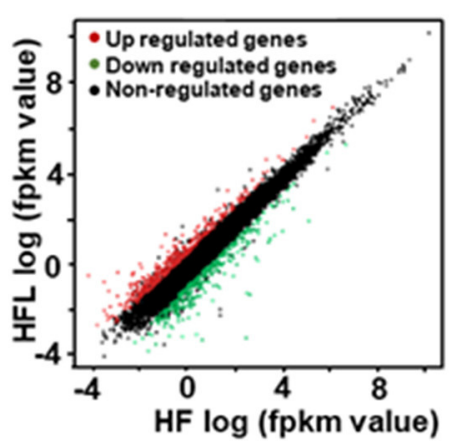

\section{DEG stat}

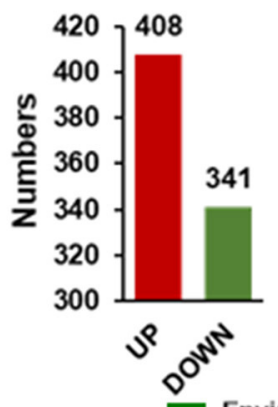

C

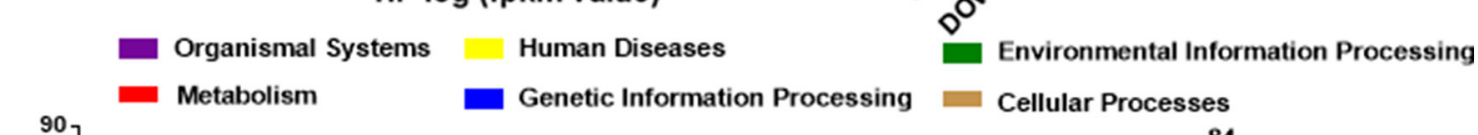

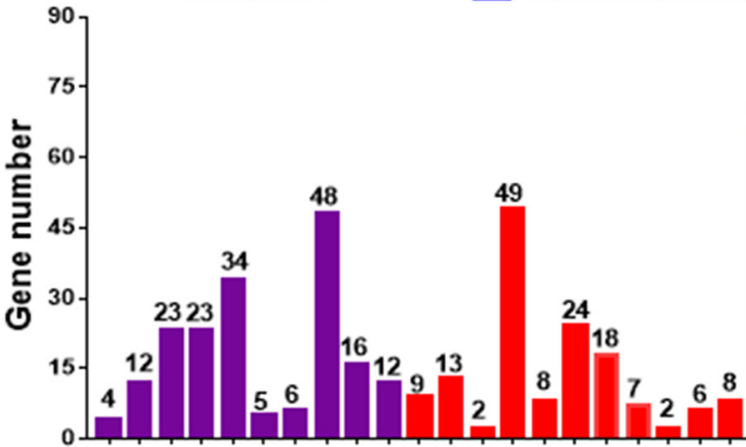

66
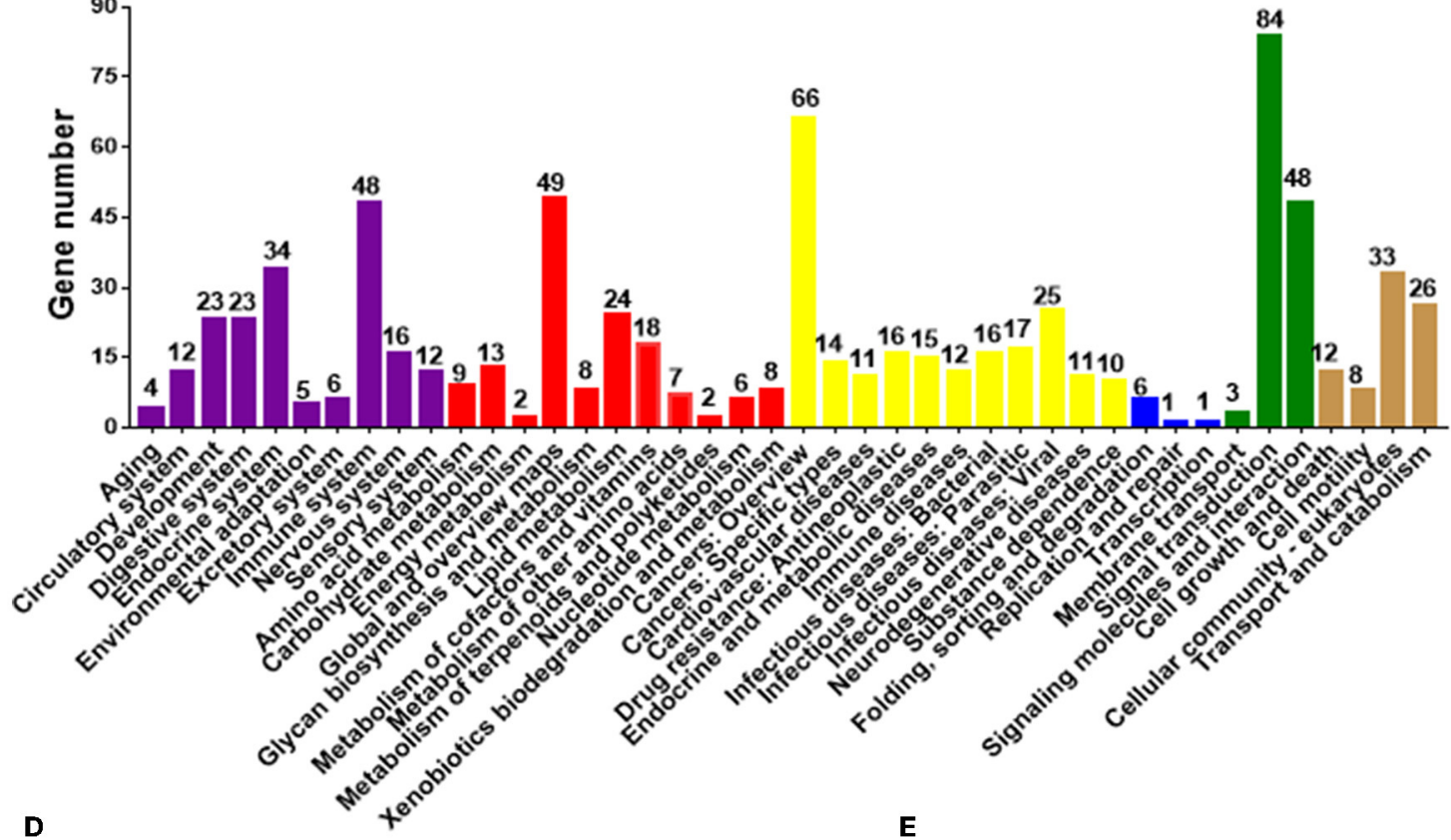

D

Immune system
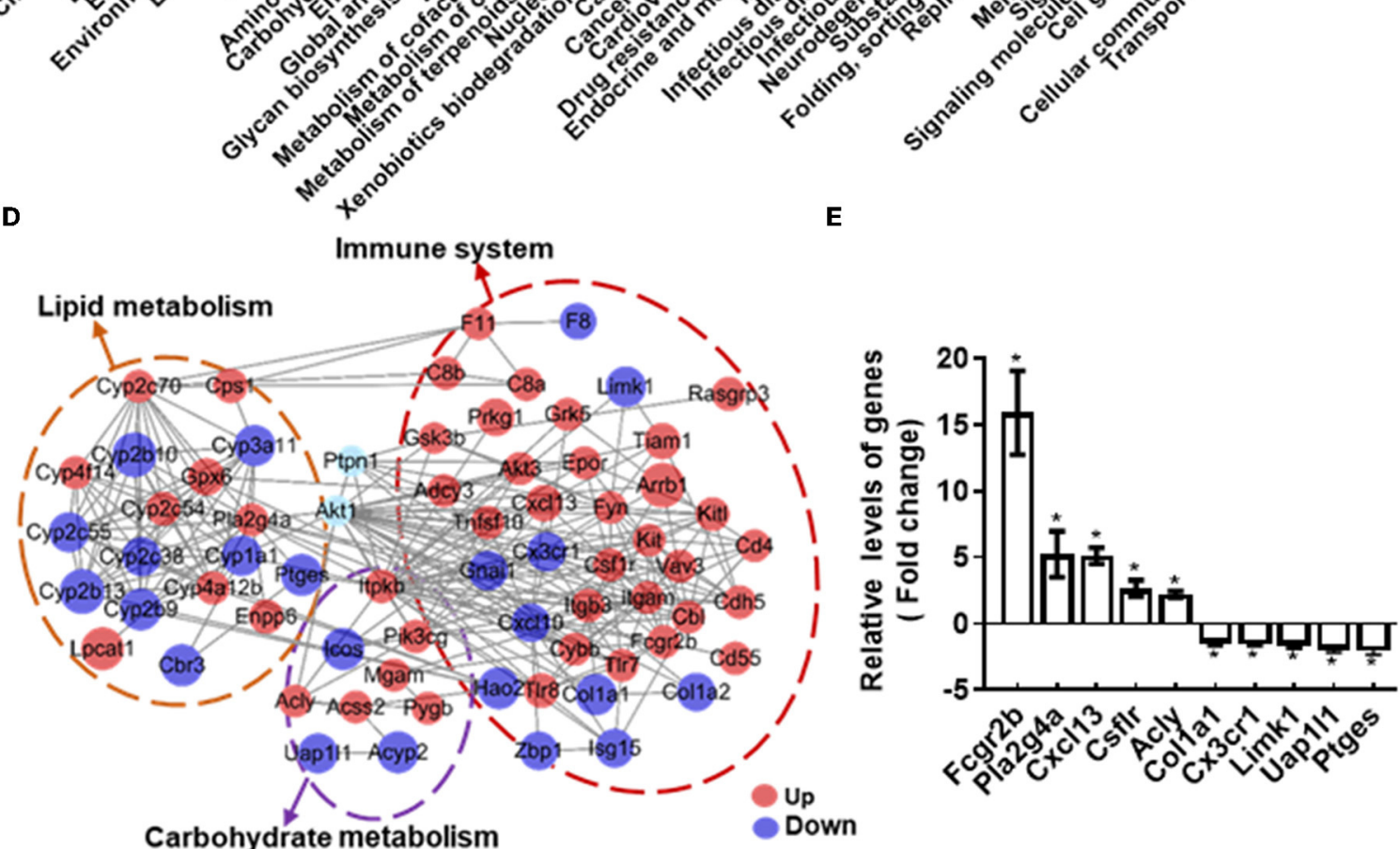

FIGURE 6 | Lentinan modulated hepatic transcriptome for immune and metabolism. (A) Scatter plots of genes in pairwise of the HFL group vs. the HF group. The $x$-axis and $y$-axis present gene expression levels. (B) The number of differentially expressed genes (DEGs). (C) KEGG pathway classification of DEGs for the HFL

group vs. the HF group. All second-level categories are grouped in top categories in different colors $(n=3)$. (D) PPI network by string analysis of DEGs involved in lipid (Continued) 
FIGURE 6 | metabolism, carbohydrate metabolism, immune system, and PTP1B-Akt signaling. The line indicates interaction evidence of nodes. Orange represents upregulation, and blue represents downregulation in the HFL mice by comparison with the HF mice. (E) qPCR analysis of the mRNA of some altered genes in the PPI network. Values are means $\pm \operatorname{SEM}(n=6) .{ }^{*} p<0.05$, vs. HF mice.

study of 24,236 adults), a high intake of mushrooms is associated with a low prevalence of NAFLD (50). However, the detailed mechanisms of mushroom in improving NAFLD have not been investigated. Here, importantly, our findings of lentinan (the main ingredient of mushroom) in benefits to the gut-liver axis suggest that lentinan as a prebiotic contributes to mushroom consumption in preventing western-style diets induced NAFLD.

Previous clinical studies have reported that the richness and diversity of gut microbiota are decreased in patients with NAFLD (51, 52). Furthermore, at the phylum level, the proportion of Bacteroidetes is decreased in patients with NAFLD (53). The progression of NAFLD is correlated with an increase in Proteobacteria (52). In this study, in the HF diet-induced NAFLD mouse model, we found that both the richness and the diversity were decreased in the gut microbiota. At phylum levels, the abundance of Bacteroidetes was decreased, whereas the Proteobacteria was increased in HF diet-fed mice. Therefore, the findings of our mouse study further suggest that overconsumption of dietary fat contributes to gut microbiota dysbiosis (in richness, diversity, and composition) in the NAFLD. Importantly, in this study, supplementation of lentinan prevented HF diet-induced gut microbiota alteration in richness and composition. At phylum levels, lentinan ameliorated the elevation of the Proteobacteria and Epsilonbacteraeota induced by a HF diet. Moreover, lentinan increased the abundance of Actinobacteria and Firmicutes at phylum levels. With LDA analysis, lentinan supplementation promoted bacteria that belongs to the phylum Actinobacteria, including order Bifidobacteriales, family Bifidobacteriaceae, and genus Bifidobacterium. These bacteria have been reported to be probiotics to improve lipid metabolism and inhibit the progression of NAFLD $(54,55)$. Therefore, lentinan in improving the gut microbiota richness and composition in the above probiotics may contribute to the prevention of NAFLD induced by the HF diet.

In this study, lentinan supplementation promoted the probiotics for gut health, such as genus Bifidobacterium. It is reported that microbiomes belonging to the genus Bifidobacterium are associated with decreased intestinal permeability of infants (56), and the administration of Bifidobacterium infantis increases the tight junction proteins, occludin and claudin four, in the intestine of a neonatal mouse model of necrotizing enterocolitis (57). The probiotics in Bifidobacterium improving gut integrity have been considered due to their potentials in reducing oxidative stress. For example, Bifidobacterium lactis HN019 supplemented in milk reduces nitric oxide metabolites levels in patients with metabolic syndrome (58). A yogurt enriched with Bifidobacterium lactis decreases malondialdehyde (a prooxidant biomarker) in patients with diabetes mellitus type two (59). Importantly, we found that lentinan supplementation significantly attenuated the alterations in oxidative stress marker iNOS and antioxidants Nrf2, HO-1, NQO1, and Gclc in the small intestinal tissue induced by the chronic HF diet. Overall, these findings suggest that lentinan supplementation in promoting probiotics, such as genus Bifidobacterium, contributes to attenuating cellular redox imbalance and intestinal barrier disruption.

In this study, we found a dramatic reduction in tight junction proteins (occludin and $\mathrm{ZO}-1$ ) in the jejunum of $\mathrm{HF}$ mice. It is reported that the intensity of $\mathrm{ZO}-1$ staining is significantly lower in the duodenum of patients with NAFLD with increased intestinal permeability (60). The abnormality of intestine permeability is related to the increased bacterial overgrowth prevalence in the small intestine of these patients (60). Interestingly, in this study, we revealed that the bacteria of phylum Proteobacteria and Epsilonbacteraeota were significantly increased in the small intestine of NAFLD mice induced by the $\mathrm{HF}$ diet. Proteobacteria is a major source of translocated antigen LPS from the gut $(61,62)$. It is reported that LPS exposure causes oxidative stress in the gut (63). NO/iNOS system mediates LPS-induced barrier dysfunction in the intestine, evidenced by that the administration of NOS inhibitor attenuates LPS-induced tight junction disruption in the ileum and colon of mice (9). Therefore, the increased Proteobacteria and its derived LPS may contribute to gut barrier dysfunction and redox imbalance. In addition, some bacteria of phylum Epsilonbacteraeota, such as order Campylobacterales, genus Campylobacter, and genus Helicobacter, are tolerant of bile acids and promote hepatic inflammation (64). Flagellins and other 19 proteins of Campylobacter jejuni are associated with the bile adaptation (65). The intragastrical inoculation of Helicobacter hepaticus induces chronic hepatitis and fibrosis in mice with the activation of p65 in the liver (66). Therefore, the above findings suggest that the bile resistance property of bacteria in phylum Epsilonbacteraeota may allow them to colonize the bile and liver to promote chronic hepatitis in the NAFLD mice induced by the HF diet. Importantly, we found that lentinan supplementation improved tight junction proteins (occludin and ZO-1) deficits in the small intestine and concurrently decreased the proportion of phylum Proteobacteria and Epsilonbacteraeota and serum LPS in HF diet-fed mice. Therefore, lentinan in the prevention of the dysbiosis of Proteobacteria, overtranslocation of its derived metabolite LPS into blood circulation and the inhibition of bileresistant Epsilonbacteraeota may be involved in the improvement of gut barrier dysfunction and hepatic inflammation induced by chronic HF diet.

The liver is an important immunological organ, in which the immune system is activated after exposure to gut microbiomederived factors, such as LPS $(13,67)$. We found that LPSbinding protein and cell surface pattern recognition receptor, Lbp and Tlr4, were significantly increased in the liver of mice fed the HF diet. Consistently, in a clinical study, Tlr4 mRNA 


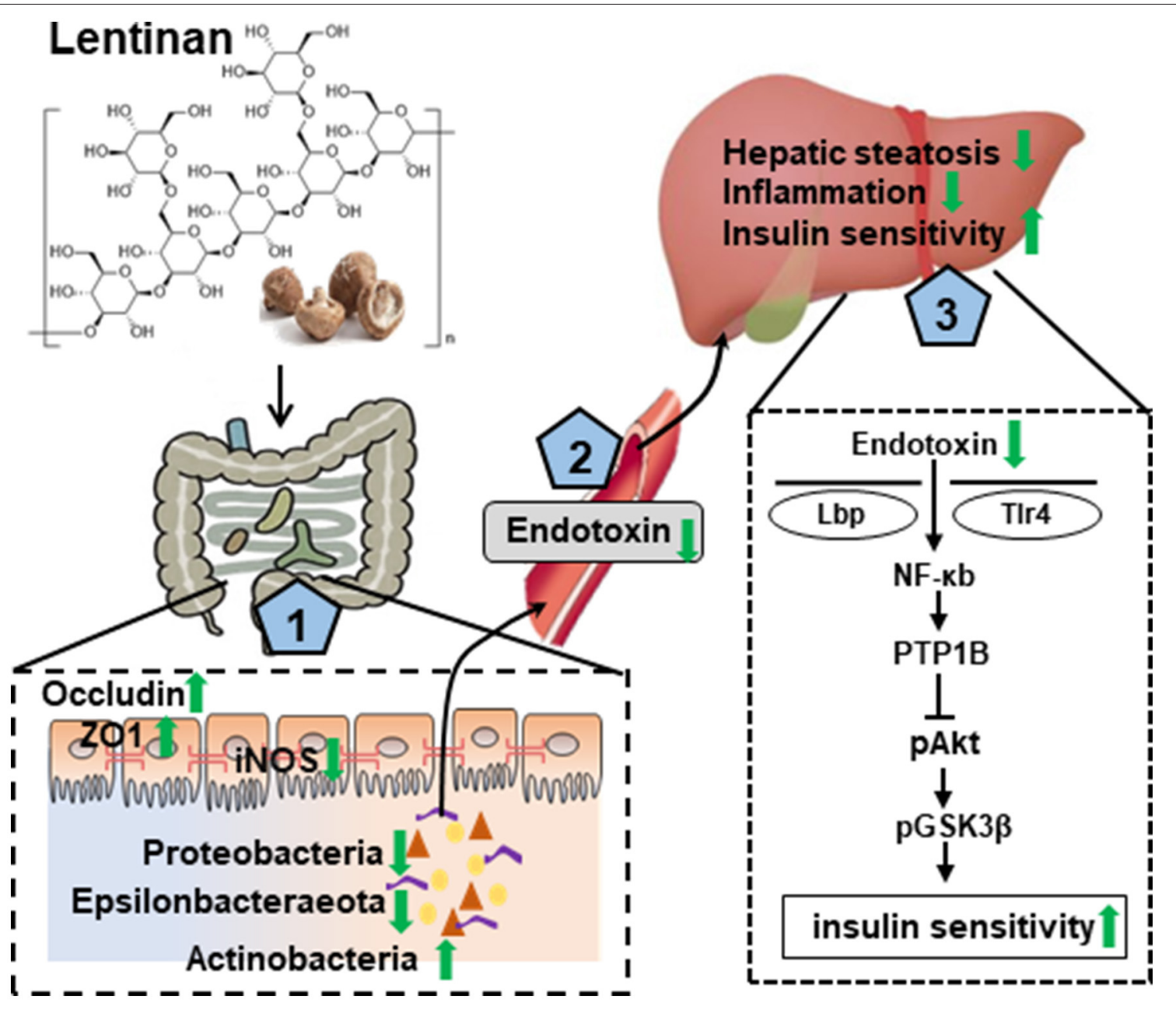

FIGURE 7 | Graphical summary of the protective effect of lentinan on the development of NAFLD via the gut-liver axis. Lentinan showed conservation of appropriate intestinal microbiota composition, resetting cellular redox balance (activation of antioxidants and inhibition of iNOS), and increasing tight junction proteins for gut epithelial integrity (1), which contribute to reduce endotoxin translocation into circulation and liver (2) and thereby to mitigate the adverse consequences of HF consumption, that is, hepatic steatosis and inflammation, and insulin resistance (3). Green arrows represent lentinan's effects.

expression is upregulated in hepatic biopsy tissue of patients with NASH with increased serum LPS (17). Tlr4 triggers the initial activation of the $N F \kappa B$ signaling pathway, which is necessary for the transcription of proinflammatory cytokines (TNF $\alpha$, IL-6, and IL-1 $\beta$ ) and overexpression of PTP1B (the inhibitor of insulin signaling) $(15,68)$. In this study, we found that the HF diet promoted steatohepatitis and glucose intolerance in mice, which had M1 proinflammatory macrophage polarization, elevated NFKB and PTP1B level and impaired insulin Akt-GSK3 $\beta$ signaling pathway in the liver. Importantly, supplementation with lentinan attenuated these adverse effects induced by the HF diet. Furthermore, the RNA-seq profile of the liver transcriptome followed by the KEGG pathways analysis also confirmed that the PI3K-Akt signaling pathway and cytokine-cytokine receptor interaction in the liver were significantly affected by lentinan. Consistently, the PPI network analysis showed that PTP1B and Akt1 are vital nodes in the network among metabolism and the immune system. In addition, the KEGG pathway analysis suggests that lentinan significantly modulated the arachidonic acid metabolism pathway, which is reported to be involved in the development and progression of NAFLD (69). However, the detailed mechanism of the arachidonic acid pathway involving lentinan improving NAFLD requires further investigation. Furthermore, according to the current mice study, supplementation of $368 \mathrm{mg}$ lentinan per day may attenuate the NAFLD in humans. However, clinical trials would be required to determine the optimum dose in future human studies.

\section{CONCLUSION}

In summary, this study with the NAFLD mouse model induced by the HF diet, chronic lentinan supplementation preserved the conservation of gut microbiota in richness and appropriate composition. Remarkably, lentinan supplementation promoted genus Bifidobacterium (important for intestinal barrier integrity 
and redox balance) and inhibited the proliferation and growth of bacteria belonging to the phylum Proteobacteria (a major source of translocated antigen LPS) and bileresistant Epsilonbacteraeota. Lentinan supplementation enhanced tight junction proteins and reset cellular redox balance (activation of antioxidants and inhibition of iNOS) in the small intestine and also improved steatohepatitis and NFкB-PTP1B-Akt-GSK3 $\beta$ (inflammation-insulin) signaling pathway in the liver. In addition to highlight the mechanisms underlying lentinan in improving NAFLD through the gut-liver axis (Figure 7), the findings of this study suggest increasing intake of mushroom ingredient, lentinan, could be a plausible strategy to mitigate the adverse effects of western-style diets on the gut microbiota and gut-liver axis in NAFLD.

\section{DATA AVAILABILITY STATEMENT}

RNA-Seq analysis raw reads were deposited into the NCBI Sequence Read Archive (SRA) database, accession number PRJNA784639. All 16S rRNA raw data were submitted to the NCBI Sequence Read Archive (SRA) database with the accession number PRJNA784743.

\section{ETHICS STATEMENT}

The animal study was reviewed and approved by the Ethics Committee of Xuzhou Medical University.

\section{REFERENCES}

1. Musso G, Cassader M, Gambino R. Non-alcoholic steatohepatitis: emerging molecular targets and therapeutic strategies. Nat Rev Drug Discov. (2016) 15:249-74. doi: 10.1038/nrd.2015.3

2. Tiniakos DG, Vos MB, Brunt E.M. Nonalcoholic fatty liver disease: pathology and pathogenesis. Annu Rev Pathol. (2010) 5:145-71. doi: 10.1146/annurev-pathol-121808-102132

3. Tripathi A, Debelius J, Brenner DA, Karin M, Loomba R, Schnabl B, et al. The gut-liver axis and the intersection with the microbiome. Nat Rev Gastroenterol Hepatol. (2018) 15:397-411. doi: 10.1038/s41575-0180011-z

4. Schnabl DA. Interactions between the intestinal microbiome and liver diseases. Gastroenterology. (2014) 146:151324. doi: 10.1053/j.gastro.2014.01.020

5. Mokhtari Z, Gibson DL, Hekmatdoost A. Nonalcoholic fatty liver disease, the gut microbiome, and diet. Adv Nutr. (2017) 8:240-52. doi: 10.3945/an.116.013151

6. Sellmann C, Degen C, Jin CJ, Nier A, Engstler AJ, Hasan Alkhatib $\mathrm{D}$, et al. Oral arginine supplementation protects female mice from the onset of non-alcoholic steatohepatitis. Amino Acids. (2017) 49:121525. doi: 10.1007/s00726-017-2423-4

7. Grishin A, Bowling J, Bell B, Wang J, Ford HR. Roles of nitric oxide and intestinal microbiota in the pathogenesis of necrotizing enterocolitis. J Pediatr Surg. (2016) 51:13-7. doi: 10.1016/j.jpedsurg.2015.10.006

8. Rao R. Oxidative stress-induced disruption of epithelial and endothelial tight junctions. Front Biosci. (2008) 13:7210-26. doi: 10.2741/3223

9. Han X, Fink MP, Yang R, Delude RL. Increased iNOS activity is essential for intestinal epithelial tight junction dysfunction in endotoxemic mice. Shock. (2004) 21:261-70. doi: 10.1097/01.shk.0000112346.38599.10

\section{AUTHOR CONTRIBUTIONS}

XY and YY: conceptualization and funding acquisition. MZhe: methodology and data curation. XG, MZho, LZ, XL, ML, and HL: validation. MZhe, JZ, and NP: formal analysis. XY: investigation. XY and MZhe: writing-original draft preparation. $\mathrm{YY}$ and KZ: writing, reviewing, and editing. X-FH and YY: visualization. All authors contributed to the article and approved the submitted version.

\section{FUNDING}

The study was funded by the National Natural Science Foundation of China $(81870854,82071184$, and 81800718), the Natural Science Foundation of the Jiangsu Higher Education Institutions of China (18KJB310015 and 19KJA560003), the Natural Science Foundation of Jiangsu Province (No. BK20211055), the Jiangsu Shuangchuang Program, the Priority Academic Program Development of Jiangsu Higher Education Institutions (PAPD) in 2014, the Starting Foundation for Talents of Xuzhou Medical University (D2018006 and D2018003), and the Jiangsu Graduate Innovation Program (No. KYCX20_2469 and No. KYCX21_2637).

\section{SUPPLEMENTARY MATERIAL}

The Supplementary Material for this article can be found online at: https://www.frontiersin.org/articles/10.3389/fnut.2021. 803691/full\#supplementary-material

10. Nam ST, Hwang JH, Kim DH, Park MJ, Lee IH, Nam HJ, et al. Role of NADH: quinone oxidoreductase-1 in the tight junctions of colonic epithelial cells. BMB Reports. (2014) 47:494-9. doi: 10.5483/BMBRep.2014.47.9.196

11. Zhang S, Zhou Q, Li Y, Zhang Y, Wu Y. MitoQ modulates lipopolysaccharideinduced intestinal barrier dysfunction via regulating Nrf2 signaling. Mediators Inflamm. (2020) 2020:3276148. doi: 10.1155/2020/3276148

12. Patouraux S, Rousseau D, Bonnafous S, Lebeaupin C, Luci C, Canivet CM, et al. CD44 is a key player in non-alcoholic steatohepatitis. J Hepatol. (2017) 67:328-38. doi: 10.1016/j.jhep.2017.03.003

13. Carpino G, Del Ben M, Pastori D, Carnevale R, Baratta F, Overi D, et al. Increased liver localization of lipopolysaccharides in human and experimental NAFLD. Hepatology. (2020) 72:470-85. doi: 10.1002/hep.31056

14. Zhu J, Zhang Y, Shen Y, Zhou H, Yu X. Lycium barbarum polysaccharides induce toll-like receptor 2- and 4-mediated phenotypic and functional maturation of murine dendritic cells via activation of NF-кB. Mol Med Rep. (2013) 8:1216-20. doi: 10.3892/mmr.2013.1608

15. Hiroki T, Shino T, Kanae K, Yohei K, Sao K, Ippo U, et al. Lipopolysaccharide (LPS)-binding protein stimulates CD14-dependent toll-like receptor 4 internalization and LPS-induced TBK1-IKK€-IRF3 axis activation. J Biol Chem. (2018) 293:10186-201. doi: 10.1074/jbc.M117.796631

16. Pardo V, González-Rodríguez Á, Guijas C, Balsinde J M, Valverde Á. Opposite cross-talk by oleate and palmitate on insulin signaling in hepatocytes through macrophage activation. J Biol Chem. (2015) 290:1166377. doi: 10.1074/jbc.M115.649483

17. Sharifnia T, Antoun J, Verriere TGC, Suarez G, Wattacheril J, Wilson KT, et al. Hepatic TLR4 signaling in obese NAFLD. Am J Physiol Gastrointest Liver Physiol. (2015) 309:G270-8. doi: 10.1152/ajpgi.00304.2014

18. Wasser SP. Medicinal mushrooms as a source of antitumor and immunomodulating polysaccharides. Appl Microbiol Biotechnol. (2002) 60:258-74. doi: 10.1007/s00253-002-1076-7 
19. Zhang S, Gu Y, Lu M, Fu J, Zhang Q, Liu L, et al. Association between edible mushroom intake and the prevalence of newly diagnosed non-alcoholic fatty liver disease: results from the TCLSIH Cohort Study in China. Br J Nutr. (2019) 121:1-8. doi: 10.1017/S000711451800301X

20. Tamura K, Hemsworth GR, Déjean G, Rogers TE, Pudlo NA, Urs K, et al. Molecular mechanism by which prominent human gut bacteroidetes utilize mixed-linkage beta-glucans, major health-promoting cereal polysaccharides. Cell Rep. (2017) 21:417-30. doi: 10.1016/j.celrep.2017.09.049

21. Butt MS, Tahir-Nadeem M, Khan MKI, Shabir R, Butt MS. Oat: unique among the cereals. Eur J Nutr. (2008) 47:68-79. doi: 10.1007/s00394-008-0698-7

22. Wang Y, Ames NP, Tun HM, Tosh SM, Jones PJ, Khafipour E. High molecular weight barley $\beta$-glucan alters gut microbiota toward reduced cardiovascular disease risk. Front Microbiol. (2016) 7:129. doi: 10.3389/fmicb.2016.00129

23. Wang $\mathrm{X}$, Wang $\mathrm{W}$, Wang L, Yu C, Zhang G, Zhu H, et al. Lentinan modulates intestinal microbiota and enhances barrier integrity in a piglet model challenged with lipopolysaccharide. Food Funct. (2019) 10:47989. doi: 10.1039/C8FO02438C

24. de Sales Guilarducci J, Marcelino B, Konig I, Orlando T, Varaschin M, Pereira LJD, et al. Accharomyces cerevisiae therapeutic effects of different doses of prebiotic (isolated from S) in comparison to $n-3$ supplement on glycemic control, lipid profiles and immunological response in diabetic rats. Diabetol Metab Syndr. (2020) 12:69. doi: 10.1186/s13098-020-00576-6

25. Reagan-Shaw S, Nihal M. Dose translation from animal to human studies revisited. FASEB J. (2008) 22:659-61. doi: 10.1096/fj.07-9574LSF

26. Liu Y, Fu X, Lan N, Li S, Zhang J, Wang S, et al. Luteolin protects against high fat diet-induced cognitive deficits in obesity mice. Behav Brain Res. (2014) 267:178-88. doi: 10.1016/j.bbr.2014.02.040

27. Huang S, Pang L, Wei CJ. Identification of a four-gene signature with prognostic significance in endometrial cancer using weighted-gene correlation network analysis. Front Genet. (2021) 12:678780. doi: 10.3389/fgene.2021.678780

28. Dennis G, Sherman B, Hosack D, Yang J, Gao W, Lane H, et al. DAVID: database for annotation, visualization, integrated discovery. Genome Biol. (2003) 4:P3. doi: 10.1186/gb-2003-4-9-r60

29. Szklarczyk D, Morris J, Cook H, Kuhn M, Wyder S, Simonovic M, et al. The STRING database in 2017: quality-controlled protein-protein association networks, made broadly accessible. Nucleic Acids Research. (2017) 45:D3628. doi: 10.1093/nar/gkw937

30. Zhou CH, Liu LL, Wu YQ, Song Z, Xing SH. Enhanced expression of salusinbeta contributes to progression of atherosclerosis in LDL receptor deficient mice. Can J Physiol Pharmacol. (2012) 90:463-71. doi: 10.1139/y2012-022

31. Martin-Venegas R, Brufau MT, Guerrero-Zamora AM, Mercier Y, Geraert PA, Ferrer R. The methionine precursor DL-2-hydroxy-(4-methylthio)butanoic acid protects intestinal epithelial barrier function. Food Chem. (2013) 141:1702-9. doi: 10.1016/j.foodchem.2013.04.081

32. Wang H, Zhu YY, Wang L, Teng T, Zhou M, Wang SG, et al. Mangiferin ameliorates fatty liver via modulation of autophagy and inflammation in high-fat-diet induced mice. Biomed Pharmacother. (2017) 96:32835. doi: 10.1016/j.biopha.2017.10.022

33. Guo M, Wu F, Hao G, Qi Q, Li R, Li N, et al. Bacillus subtilis improves immunity and disease resistance in rabbits. Front Immunol. (2017) 8:354. doi: 10.3389/fimmu.2017.00354

34. Chen S, Zhou Y, Chen Y, Gu J. Fastp: an ultra-fast allin-one FASTQ preprocessor. Bioinformatics. (2018) 34:i88490. doi: 10.1093/bioinformatics/bty560

35. Magoč T, Salzberg SL. FLASH: fast length adjustment of short reads to improve genome assemblies. Bioinformatics. (2011) 27:2957-63. doi: 10.1093/bioinformatics/btr507

36. Caporaso JG, Kuczynski J, Stombaugh J, Bittinger K, Bushman FD, Costello EK, et al. QIIME allows analysis of high-throughput community sequencing data. Nat Methods. (2010) 7:335-6. doi: 10.1038/nmeth.f.303

37. Bokulich NA, Subramanian S, Faith JJ, Gevers D, Gordon JI, Knight, et al. Quality-filtering vastly improves diversity estimates from Illumina amplicon sequencing. Nat Methods. (2013) 10:57-9. doi: 10.1038/nmeth.2276

38. Edgar RC. UPARSE: highly accurate OTU sequences from microbial amplicon reads. Nat Methods. (2013) 10:996-8. doi: 10.1038/nmet h. 2604
39. Schloss PD, Westcott SL, Ryabin T, Hall JR, Hartmann M, Hollister EB, et al. Introducing mothur: open-source, platform-independent, communitysupported software for describing and comparing microbial communities. Appl Environ Microbiol. (2009) 75:7537-41. doi: 10.1128/AEM.01541-09

40. Segata N, Izard J, Waldron L, Gevers D, Miropolsky L, Garrett WS, et al. Metagenomic biomarker discovery and explanation. Genome Biology. (2011) 12:R60. doi: 10.1186/gb-2011-12-6-r60

41. Sung JJY, Coker OO, Chu E, Szeto CH, Luk STY, Lau HCH, et al. Gastric microbes associated with gastric inflammation, atrophy and intestinal metaplasia 1 year after helicobacter pylori eradication. Gut. (2020) 69:157280. doi: 10.1136/gutjnl-2019-319826

42. Langille MG, Zaneveld J, Caporaso JG, McDonald D, Knights D, Reyes JA, et al. Predictive functional profiling of microbial communities using 16S rRNA marker gene sequences. Nat Biotechnol. (2013) 31:81421. doi: $10.1038 /$ nbt. 2676

43. Kirpich IA, Marsano LS, McClain CJ. Gut-liver axis, nutrition, and non-alcoholic fatty liver disease. Clin Biochem. (2015) 48:923-30. doi: 10.1016/j.clinbiochem.2015.06.023

44. Ni Y, Ni L, Zhuge F, Fu Z. The gut microbiota and its metabolites, novel targets for treating and preventing non-alcoholic fatty liver disease. Mol Nutr Food Res. (2020) 64:e2000375. doi: 10.1002/mnfr.202000375

45. Gomes AC, Hoffmann C, Mota JF. The human gut microbiota: metabolism and perspective in obesity. Gut Microbes. (2018) 9:308-25. doi: 10.1080/19490976.2018.1465157

46. Brenachot X, Ramadori G, Ioris R, Veyrat-Durebex C, Altirriba J, Aras E, et al. Hepatic protein tyrosine phosphatase receptor gamma links obesity-induced inflammation to insulin resistance. Nat Commun. (2017) 8:1820. doi: 10.1038/s41467-017-02074-2

47. Tian J, Liao X, Wang Y, Si X, Shu C, Gong E, et al. Identification of cyanidin-3-arabinoside extracted from blueberry as a selective protein tyrosine phosphatase 1B inhibitor. J Agric Food Chem. (2019) 67:1362434. doi: 10.1021/acs.jafc.9b06155

48. Iñiguez $M$, Pérez-Matute $P$, Villanueva-Millán MJ, Recio-Fernández E, Roncero-Ramos I, Pérez-Clavijo M, et al. Agaricus bisporus supplementation reduces high-fat diet-induced body weight gain and fatty liver development. $J$ Physiol Biochem. (2018) 74:635-46. doi: 10.1007/s13105-018-0649-6

49. Nagao K, Inoue $\mathrm{N}$, Inafuku $\mathrm{M}$, Shirouchi B, Morooka $\mathrm{T}$, Nomura $\mathrm{S}$, et al. Mukitake mushroom (Panellus serotinus) alleviates nonalcoholic fatty liver disease through the suppression of monocyte chemoattractant protein 1 production in $\mathrm{db} / \mathrm{db}$ mice. J Nutr Biochem. (2010) 21:41823. doi: 10.1016/j.jnutbio.2009.01.021

50. Zhang S, Gu Y, Lu M, Fu J, Zhang Q, Liu L, et al. Association between edible mushroom intake and the prevalence of newly diagnosed non-alcoholic fatty liver disease: results from the tianjin chronic low-grade systemic inflammation and health cohort study in China. Br J Nutr. (2020) 123:10412. doi: 10.1017/S0007114519002605

51. Kim H-N, Joo E-J, Cheong HS, Kim Y, Kim H-L, Shin H, et al. Gut microbiota and risk of persistent nonalcoholic fatty liver diseases. J Clin Med. (2019) 8:1089. doi: $10.3390 / j \mathrm{~cm} 8081089$

52. Caussy C, Tripathi A, Humphrey G, Bassirian S, Singh S, Faulkner C, et al. A gut microbiome signature for cirrhosis due to nonalcoholic fatty liver disease. Nat Commun. (2019) 10:1406. doi: 10.1038/s41467-019-09455-9

53. Mouzaki M, Comelli EM, Arendt BM, Bonengel J, Fung SK, Fischer SE, et al. Intestinal microbiota in patients with nonalcoholic fatty liver disease. Hepatology. (2013) 58:120-7. doi: 10.1002/hep.26319

54. Li C, Nie SP, Zhu KX, Ding Q, Li C, Xiong T, et al. Lactobacillus plantarum NCU116 improves liver function, oxidative stress and lipid metabolism in rats with high fat diet induced non-alcoholic fatty liver disease. Food Funct. (2014) 5:3216-23. doi: 10.1039/C4FO00549J

55. Hye AK, Jeon-Kyung K, Jae-Young K, Se-Eun J, Joo HM, DongHyun K. Lactobacillus plantarum LC27 and Bifidobacterium longum LC67 simultaneously alleviate high-fat diet-induced colitis, endotoxemia, liver steatosis, and obesity in mice. Nutr Res. (2019) 67:78-89. doi: 10.1016/j.nutres.2019.03.008

56. Underwood MA, German JB, Lebrilla CB, Mills DA. Bifidobacterium longum subspecies infantis: champion colonizer of the infant gut. Pediatr Res. (2015) 77:229-35. doi: 10.1038/pr.2014.156 
57. Bergmann KR, Liu SXL, Tian R, Kushnir A, Turner JR, Li H-L, et al. Bifidobacteria stabilize claudins at tight junctions and prevent intestinal barrier dysfunction in mouse necrotizing enterocolitis. Am J Pathol. (2013) 182:1595-606. doi: 10.1016/j.ajpath.2013.01.013

58. Bernini LJ, Simão ANC, de Souza CHB, Alfieri DF, Segura LG, Costa GN, et al. Effect of bifidobacterium lactis HN019 on inflammatory markers and oxidative stress in subjects with and without the metabolic syndrome. $\mathrm{Br} \mathrm{J}$ Nutr. (2018) 120:645-52. doi: 10.1017/S0007114518001861

59. Ejtahed HS, Mohtadi-Nia J, Homayouni-Rad A, Niafar M, Asghari-Jafarabadi M, Mofid V. Probiotic yogurt improves antioxidant status in type 2 diabetic patients. Nutrition. (2012) 28:539-43. doi: 10.1016/j.nut.2011.08.013

60. Miele L, Valenza V, La Torre G, Montalto M, Cammarota G, Ricci, et al. Increased intestinal permeability and tight junction alterations in nonalcoholic fatty liver disease. Hepatology. (2009) 49:1877-87. doi: 10.1002/hep.22848

61. Needham BD, Carroll SM, Giles DK, Georgiou G, Whiteley M, Trent MS. Modulating the innate immune response by combinatorial engineering of endotoxin. Proc Natl Acad Sci USA. (2013) 110:1464-9. doi: 10.1073/pnas.1218080110

62. Vatanen T, Kostic AD, d'Hennezel E, Siljander H, Franzosa EA, Yassour M, et al. Variation in microbiome LPS immunogenicity contributes to autoimmunity in humans. Cell. (2016) 165:84253. doi: 10.1016/j.cell.2016.04.007

63. Wu QJ, Wang YQ, Qi YX. The protective effect of procyanidin against LPSinduced acute gut injury by the regulations of oxidative state. SpringerPlus. (2016) 5:1645. doi: 10.1186/s40064-016-3306-y

64. Okoli AS, Wadstrom T, Mendz GL. Minireview: bioinformatic study of bile responses in Campylobacterales. FEMS Immunol Med Microbiol. (2007) 49:101-23. doi: 10.1111/j.1574-695X.2006.00194.x

65. Fox E, Raftery M, Goodchild A, Mendz GJ. Campylobacter jejuni response to ox-bile stress. FEMS Immunol Med Microbiol. (2007) 49:165-72. doi: 10.1111/j.1574-695X.2006. 00190.x
66. Cao S, Zhu L, Zhu C, Feng J, Yin J, Lu J, et al. Helicobacter hepaticus infection-induced IL-33 promotes hepatic inflammation and fibrosis through ST2 signaling pathways in BALB/c mice. Biochem Biophys Res Commun. (2020) 525:654-61. doi: 10.1016/j.bbrc.2020.02.139

67. Konturek PC, Harsch IA, Konturek K, Schink M, Konturek T, Neurath MF, et al. Gut ${ }^{-}$liver axis: how do gut bacteria influence the liver? Med Sci. (2018) 6:79. doi: 10.3390/medsci6030079

68. Zabolotny J, Kim Y, Welsh L, Kershaw E, Neel B, Kahn B. Protein-tyrosine phosphatase $1 \mathrm{~B}$ expression is induced by inflammation in vivo. J Biol Chem. (2008) 283:14230-41. doi: 10.1074/jbc.M800061200

69. Ma K, Chen Y, Liang X, Miao J, Zhao Q. Inhibition of 5lipoxygenase inhibitor zileuton in high-fat diet-induced nonalcoholic fatty liver disease progression model. Iran J Basic Med Sci. (2017) 20:1207-12. doi: 10.22038/IJBMS.2017.9482

Conflict of Interest: The authors declare that the research was conducted in the absence of any commercial or financial relationships that could be construed as a potential conflict of interest.

Publisher's Note: All claims expressed in this article are solely those of the authors and do not necessarily represent those of their affiliated organizations, or those of the publisher, the editors and the reviewers. Any product that may be evaluated in this article, or claim that may be made by its manufacturer, is not guaranteed or endorsed by the publisher.

Copyright $\odot 2022$ Yang, Zheng, Zhou, Zhou, Ge, Pang, Li, Li, Li, Zhang, Huang, Zheng and $Y u$. This is an open-access article distributed under the terms of the Creative Commons Attribution License (CC BY). The use, distribution or reproduction in other forums is permitted, provided the original author(s) and the copyright owner(s) are credited and that the original publication in this journal is cited, in accordance with accepted academic practice. No use, distribution or reproduction is permitted which does not comply with these terms. 